\title{
Beyond three frequencies: An extendable model for single-epoch decimeter-level point positioning by exploiting Galileo and BeiDou-3 signals
}

\author{
Jianghui Geng ${ }^{1}$, Jiang Guo ${ }^{1}$ \\ 1 GNSS Research Center, Wuhan University \\ E-mail: jgeng@whu.edu.cn; guojiang@,whu.edu.cn
}

\begin{abstract}
GNSS is indispensable to self-driving vehicles by delivering decimeter-level or better absolute positioning solutions. Such a high precision normally requires a convergence time spanning seconds to minutes, which is however unrealistic in extremely difficult driving conditions where GNSS signals are obstructed frequently. Such convergences, no matter how short, will greatly risk and discredit autonomous driving in satisfying stringent life-safety standards. In this study, we therefore developed an extendable GNSS precise point positioning (PPP) model to exploit the advanced Galileo/BeiDou3 more-than-three-frequency signals with the goal of achieving instant or single-epoch 10-30 $\mathrm{cm}$ positioning accuracy and over $99 \%$ availability for the horizontal components over wide areas. In particular, uncombined Galileo/BeiDou-3 signals on all available frequencies were injected simultaneously into PPP to perform singleepoch wide-lane ambiguity resolution (PPP-WAR) after phase bias calibrations on raw observations. Experimenting on the Galileo five-frequency data from 36 stations in Australia, we found that instant PPP-WAR was accomplished at more than $99.5 \%$ of all epochs; we achieved an instant positioning accuracy of 0.10 and $0.11 \mathrm{~m}(1 \sigma)$ for the east and north components, respectively, using Galileo E1/E5a/E5/E5b/E6 signals from less than 10 satellites, while 0.16 and $0.23 \mathrm{~m}$ using BeiDou-3 B1C/B1I/B2a/B2b/B3I signals from only 5-6 satellites per epoch observed by 10 stations within China. Moreover, we carried out vehicle-borne experiments collecting multi-frequency Galileo/BeiDou-3 signals in case of overpass and tunnel adversities. With 7 Galileo/BeiDou-3 satellites per epoch on average, instant PPP-WAR reached a mean positioning accuracy of 0.23 and $0.24 \mathrm{~m}$ for the horizontal components, which
\end{abstract}


can be further improved to 0.14 and $0.12 \mathrm{~m}$ when multi-epoch filtering is preferably enabled. More encouragingly, though this positioning accuracy can also be ensured with triple-frequency data, the data redundancy favored by even more frequencies can reduce the high-precision recovery time from up to $4 \mathrm{~s}$ to $2 \mathrm{~s}$ in case of total signal blockages. With the rapidly ongoing deployment of Galileo, BeiDou-3 and other GNSS constellations, we can envision an instant global positioning service characterized by around $20-\mathrm{cm}$ horizontal accuracy and over $99 \%$ availability for selfdriving vehicles.

Keywords Instant decimeter-level point positioning; Five-frequency; BeiDou-3; Galileo E6; Precise Point Positioning; Ambiguity resolution

\section{Introduction}

GNSS has been recognized as an indispensable component to navigate self-driving vehicles safely. Autonomous driving demands high positioning accuracy as well as, more critically, high position availability. On the one hand, decimeter-level accuracy is required for lane recognition while an even 10-cm accuracy is desired for the trajectory maintenance and pedestrian avoidance, for example (Schreiber et al., 2013). On the other hand, accurate positions of high availability are life-critical since any lack of credible locations will jeopardize and risk unsupervised vehicles driving in complex urban environments; with regard to GNSS, such "high availability" is best ensured by exploiting both multi-constellation and multi-frequency satellite signals to achieve instant or single-epoch high-precision positioning (e.g., Geng et al., 2019a).

Conventionally, instant centimeter-level positioning is securely achieved on ultra-short baseline (e.g., <2 km) solutions (e.g., Odolinski et al., 2015; Prochniewicz et al., 2016; Teunissen et al., 2014). Such stringent requirement on ultra-short baselines could be relaxed in part in case of network RTK (e.g., Kim et al., 2017; Stephenson et al., 2013), but it will be impractical to maintain a dense reference 
network over vast areas. In contrast, single-epoch positioning over longer baselines (e.g., up to $100 \mathrm{~km}$ ) serving wide areas was attempted using triple-frequency GNSS, despite the degradation of positioning precision to the decimeter level. In particular, Feng and Li (2010) prototyped an idea of resolving two wide-lane combination observables only for ultra-rapid decimeter-level positioning over medium to long baselines (about $100 \mathrm{~km}$ ), which was later revisited by Li et al. (2017) using real BeiDou-2 triple-frequency data. Since these developments still require relatively dense reference networks, precise point positioning (PPP) seems preferred for global applications (Zumberge et al. 1997), though its ambiguity resolution (PPP-AR) efficiency is far poorer than those functioning on short baselines (Geng et al., 2011).

To be specific, PPP-AR has been constantly suffering from long convergence times of up to a few tens of minutes. Such convergence or initialization times are usually contingent upon satellite geometry and pseudorange precisions (Geng et al., 2011). To speed up convergences, Nadarajah et al. (2018) carried out dual-frequency PPP-AR by integrating GPS, BeiDou and Galileo data and finally achieved a mean convergence time of 15 minutes. On the contrary, Li et al. (2018) implemented BeiDou-only PPP-AR using triple-frequency data and decreased the convergence time by $10 \%$ compared to that of dual-frequency ambiguity-fixed solutions. Using triplefrequency GPS/BeiDou/Galileo/QZSS (Quasi-Zenith Satellite System) data, ultimately, Geng et al. (2019b) achieved PPP-AR with a mean time of about 6 minutes. However, such a few minutes of convergence time still does not fit autonomous driving demanding super high availability of accurate positions, especially in urban valleys where loss of satellite signals takes place frequently.

The bottleneck of shortening PPP-AR convergence times boils down to the inefficient narrow-lane ambiguity fixing. Narrow-lane ambiguities have a wavelength of only $10 \mathrm{~cm}$, and thus can hardly converge fast in case of the pseudorange with decimeter-level noise. By ignoring narrow-lane ambiguity fixing intentionally, Geng and Bock (2013) found that instant PPP might be achievable by fixing only 
(extra-)wide-lane ambiguities using simulated triple-frequency GPS data, despite the sacrifice of amplifying the wide-lane phase noise by over 100 times as well as degrading the positioning precision from the centimeter to decimeter level. However, this trade-off between the positioning efficiency and accuracy can open up the feasibility of applying triple-frequency PPP to self-driving vehicles. Later, Geng et al. (2019a) improved this experiment by introducing real triple-frequency

GPS/BeiDou/Galileo/QZSS data into uncombined PPP to resolve only (extra-)widelane ambiguities, which is named as PPP-WAR to highlight single-receiver wide-lane ambiguity resolution. They showed that a positioning accuracy of around $0.3 \mathrm{~m}$ in the horizontal direction can be reached using a single epoch of data with a success rate of over $90 \%$. However, there are still two prime concerns for PPP-WAR to pillar autonomous driving or other safety-critical applications. First, any loss of the thirdfrequency signal will negate PPP-WAR and consequently instant decimeter-level positions will be unavailable. Second, vehicle collision avoidance in case of crowded traffic desires an instant positioning accuracy of about $10-30 \mathrm{~cm}$ over $>99 \%$ of epochs, which is still challenging for the PPP-WAR solutions realized by Geng et al. (2019a) even though over 10 satellites were visible per epoch.

Table 1 Characteristics of BeiDou-3 open service signals (Lu et al., 2019; Zhang et al., 2017)

\begin{tabular}{lllll}
\hline Band & Component & Frequency $(\mathrm{MHz})$ & Modulation & Code Rate (Mcps) \\
\hline B1I & & 1561.098 & $\operatorname{BPSK}(2)$ & 2.046 \\
\hline B1C & B1C_data & 1575.42 & $\operatorname{BOC}(1,1)$ & 1.023 \\
& B1C_pilot & & QMBOC $(6,1,4 / 33)$ & \\
\hline B2a & B2a_data & 1176.45 & QPSK $(10)$ & 10.23 \\
& B2a_pilot & & & 10.23 \\
\hline B2b & B2b_data & 1207.14 & QPSK(10) & \\
\hline B3I & B2b_pilot & & & 10.23 \\
\hline
\end{tabular}


Fortunately, the advanced designs of multi-frequency signals from Galileo and BeiDou-3 (e.g., Galileo E1/E5a/E5/E5b/E6 and BeiDou-3 B1C/B1I/B2a/B2b/B3I, Table 1) bring new opportunities for instant PPP-WAR by improving both positioning accuracy and availability. For instance, by virtue of Galileo E1/E5a/E6 signals for PPP-WAR, Laurichesse and Banville (2018) allegedly achieved instant 20-cm point positioning in the horizontal component with only 7 satellites visible. More comprehensively, Guo and Xin (2019) showed that Galileo E1/E5a/E6 PPP-WAR outperformed its E1/E5a/E5b counterpart since the wide-lane phase noise was amplified by only 67 instead of 172 times (cf., Hatch 2006). More favorably, BeiDou3 B1C/B2a/B3I signals have the similar trait of enabling instant PPP-WAR by suppressing the wide-lane phase noise amplification down to only 71 times, which is expected to lead to a positioning accuracy commensurate with those based on Galileo E1/E5a/E6. In the meantime, different from GPS and QZSS signals that are modulated following the Binary/Quadrature Phase Shift Keying (BPSK \& QPSK) strategies, both BeiDou-3 and Galileo signals have adopted the Binary Offset Carrier (BOC) modulation schemes, which results in a capability of mitigating multipath effects and tracking errors significantly for code/phase observables (Fantino et al., 2008). It is therefore a promising trial to integrate Galileo and BeiDou-3 to enable PPP-WAR for improved positioning instantaneity and accuracy. This idea was also inspected by Wang et al. (2018) where a 7\% rise of the success rates was achieved when Galileo five-frequency, instead of triple-frequency, ambiguity resolution was attempted over long baselines (200-400 km). In this case, we can also incorporate Galileo E5b/E5 signals into E1/E5a/E6-enabled PPP-WAR (BeiDou-3 similarly) with the motivation of improving ambiguity resolution efficiency; moreover, the data redundancy favored by five-frequency signals is able to ensure high position availability in case of loss of signal tracking.

In this study, we therefore proposed an extendable PPP-WAR model being able to ingest more than three frequencies of carrier-phase data towards instant $10-30 \mathrm{~cm}$ 
horizontal positioning accuracy with super high availability. Especially, we will exploit Galileo E1/E5a/E6 and BeiDou-3 B1C/B2a/B3I signals in addition to E5/E5b and B1I/B2b signals. The study is organized as follows: Section 2 demonstrates the extendable PPP-WAR model based on uncombined PPP; section 3 details the data processing strategy for static stations and vehicle-borne experiments; section 4 delineates the positioning performance of instant multi-frequency PPP-WAR while section 5 in turn discusses how it depends on the noise level of wide-lane observables; section 6 draws conclusions.

\section{Methods}

GNSS multi-frequency raw observation models for pseudorange and carrier-phase in the unit of length can be written as

$$
\left\{\begin{array}{l}
P_{i, j}^{k}=\rho_{i}^{k}+g_{s, j}^{2} \gamma_{i}^{k}+c\left(t_{i}-t^{k}\right)+d_{i, j}^{s}-d_{j}^{k} \\
L_{i, j}^{k}=\rho_{i}^{k}-g_{s, j}^{2} \gamma_{i}^{k}+c\left(t_{i}-t^{k}\right)+\lambda_{s, j}\left(N_{i, j}^{k}+b_{i, j}^{s}-b_{j}^{k}\right)
\end{array}\right.
$$

where $P_{i, j}^{k}$ and $L_{i, j}^{k}$ denote the pseudorange and carrier-phase on frequency $j$ $(j=1,2, \ldots n)$ between satellite $k$ and station $i ; \rho_{i}^{k}$ is the geometry distance which also includes atmosphere delays, phase wind-up and relativistic effects; $s$ can be either " $E$ " for Galileo or " $C$ " for BeiDou-3; $\gamma_{i}^{k}$ denotes the ionosphere delay and $g_{s, j}^{2}$ is the frequency-dependent scaling coefficient; in particular, $g_{s, j}$ equates $\frac{f_{s, 1}}{f_{s, j}}$ where $f_{s, j}$ is the frequency of carrier-phase $j ; c$ is the speed of light in vacuum and $\lambda_{s, j}=\frac{c}{f_{s, j}}$ is the wavelength; $t_{i}$ and $t^{k}$ denote the physical clock errors for station $i$ and satellite $k$, respectively; $d_{i, j}^{s}$ and $d_{j}^{k}$ denote the frequency-dependent pseudorange hardware biases at station $i$ and satellite $k$, respectively, while $b_{i, j}^{s}$ and $b_{j}^{k}$ are those for carrier-phase, all of which are 
presumed time-constant throughout for brevity of mathematical derivations; $N_{i, j}^{k}$ represents the integer ambiguity.

However, all unknowns in Eq. 1 cannot be solved simultaneously owing to the linear dependency between clocks, hardware bias and ambiguity parameters. Hence, we reformulate Eq. 1 where the hardware biases are lumped into the clock parameters (cf. de Jonge, 1998; Odijk et al., 2016), that is

$$
\left\{\begin{array}{l}
P_{i, 1}^{k}=\rho_{i}^{k}+\bar{\gamma}_{i}^{k}+\bar{t}_{i}^{s}-\bar{t}^{k} \\
P_{i, 2}^{k}=\rho_{i}^{k}+g_{s, 2}^{2} \bar{\gamma}_{i}^{k}+\bar{t}_{i}^{s}-\bar{t}^{k} \\
P_{i, j}^{k}=\rho_{i}^{k}+g_{s, j}^{2} \bar{\gamma}_{i}^{k}+\bar{t}_{i}^{s}-\bar{t}^{k}+h_{i, j}^{k} \\
L_{i, 1}^{k}=\rho_{i}^{k}-\bar{\gamma}_{i}^{k}+\bar{t}_{i}^{s}-\bar{t}^{k}+\lambda_{s, 1} \bar{N}_{i, 1}^{k} \\
L_{i, 2}^{k}=\rho_{i}^{k}-g_{s, 2}^{2} \bar{\gamma}_{i}^{k}+\bar{t}_{i}^{s}-\bar{t}^{k}+\lambda_{s, 2} \bar{N}_{i, 2}^{k} \\
L_{i, j}^{k}=\rho_{i}^{k}-g_{s, j}^{2} \bar{\gamma}_{i}^{k}+\bar{t}_{i}^{s}-\bar{t}^{k}+\lambda_{s, j} \bar{N}_{i, j}^{k}
\end{array}\right.
$$

and

$$
\left\{\begin{array}{l}
\alpha=\frac{g_{s, 2}^{2}}{g_{s, 2}^{s}-1} \\
\beta=\frac{1}{g_{s, 2}^{2}-1} \\
\bar{t}_{i}^{s}=c t_{i}+\alpha d_{i, 1}^{s}-\beta d_{i, 2}^{s} \\
\bar{t}^{k}=c t^{k}+\alpha d_{1}^{k}-\beta d_{2}^{k} \\
\bar{\gamma}_{i}^{k}=\gamma_{i}^{k}-\beta\left(d_{i, 1}^{s}-d_{i, 2}^{s}-d_{1}^{k}+d_{2}^{k}\right) \\
h_{i, j}^{k}=g_{s, j}^{2} \beta\left(d_{i, 1}^{s}-d_{i, 2}^{s}\right)-\left(\alpha d_{i, 1}^{s}-\beta d_{i, 2}^{s}\right)+d_{i, j}^{s}-g_{s, j}^{2} \beta\left(d_{1}^{k}-d_{2}^{k}\right)+\alpha d_{1}^{k}-\beta d_{2}^{k}-d_{j}^{k} \\
\bar{N}_{i, 1}^{k}=N_{i, 1}^{k}+b_{i, 1}^{s}-b_{1}^{k}-\frac{1}{\lambda_{s, 1}}\left((\alpha+\beta) d_{i, 1}^{s}-2 \beta d_{i, 2}^{s}-(\alpha+\beta) d_{1}^{k}+2 \beta d_{2}^{k}\right) \\
\bar{N}_{i, 2}^{k}=N_{i, 2}^{k}+b_{i, 2}^{s}-b_{2}^{k}-\frac{1}{\lambda_{s, 2}}\left(2 \beta d_{i, 1}^{s}-(\alpha+\beta) d_{i, 2}^{s}-2 \beta d_{1}^{k}+(\alpha+\beta) d_{2}^{k}\right) \\
\bar{N}_{i, j}^{k}=N_{i, j}^{k}+b_{i, j}^{s}-b_{j}^{k}-\frac{1}{\lambda_{s, j}}\left(g_{s, j}^{2} \beta\left(d_{i, 1}^{s}-d_{i, 2}^{s}\right)+\alpha d_{i, 1}^{s}-\beta d_{i, 2}^{s}-g_{s, j}^{2} \beta\left(d_{1}^{k}-d_{2}^{k}\right)-\alpha d_{1}^{k}+\beta d_{2}^{k}\right)
\end{array}\right.
$$

where "1" and " 2 " represent the first two frequencies which are E1/E5a for Galileo and B1C/B2a for BeiDou-3, and thus $j$ denotes all remaining signals; $\bar{t}_{i}^{s}$ and $\bar{t}^{k}$ are the new receiver and satellite clock parameters, respectively; $\bar{\gamma}_{i}^{k}$ denotes the new 
ionosphere delay parameter which suffers from hardware bias contamination; $\bar{N}_{i, 1}^{k}$, $\bar{N}_{i, 2}^{k}$ and $\bar{N}_{i, j}^{k}$ are new but non-integer ambiguity parameters. Moreover, we consider that the inter-frequency clock biases (IFCBs) for Galileo and BeiDou-3 are time-constant (Zhang et al., 2017), and we therefore assume that all multi-frequency observables share the same receiver clock, satellite clock and ionosphere delay parameters by introducing an inter-frequency bias parameter $h_{i, j}^{k}$ for each pseudorange except $P_{i, 1}^{k}$ and $P_{i, 2}^{k}$; note that each $h_{i, j}^{k}$ is estimated as a daily constant.

In addition, Eq. 2 only shows the functional model for a single constellation. For an integrated Galileo/BeiDou-3 processing, we estimated a common receiver clock with an inter-system code bias estimated daily for BeiDou-3. Satellite clocks were computed over a reference network with zero-mean constraints imposed on receiver clocks and inter-system code biases (Odijk et al., 2016).

\subsection{Undifferenced uncombined phase biases}

Phase bias corrections should be computed before PPP-WAR. Ge et al. (2008) have indicated that the integer nature of single-receiver ambiguities was destroyed by uncalibrated phase delays (UPDs) or phase biases according to IGS (International GNSS Service) conventions. Phase biases can be taken theoretically as the hardware biases lumped into integer ambiguities, and thus can be computed using the raw ambiguity estimates from a reference network. Specifically, after PPP processing based on Eq. 2, with respect to the undifferenced ambiguity estimates on a reference frequency (i.e., E6 for Galileo and B3I for BeiDou-3 throughout), we converted the remaining undifferenced ambiguities into their wide-lane counterparts, that is

$$
\bar{N}_{i, j^{*}}^{k}=\bar{N}_{i, j}^{k}-\bar{N}_{i, *}^{k}
$$


where "*" denotes the reference frequency and $j$ represents all other frequencies processed in Eq. 2.

The wide-lane ambiguities in Eq. 4 are not integers in nature since comprising non-integer wide-lane phase biases. Since receiver-dependent phase biases cannot be separated from their satellite-dependent counterparts, they are usually eliminated by differencing ambiguities between satellites, and then we obtain

$$
\bar{N}_{i, j^{*}}^{k q}=\overline{\bar{N}}_{i, j^{*}}^{k q}-\bar{b}_{j^{*}}^{k q}
$$

where

$$
\left\{\begin{array}{c}
\bar{N}_{i, j^{*}}^{k q}=\bar{N}_{i, j^{*}}^{k}-\bar{N}_{i, j^{*}}^{q} \\
\overline{\bar{N}}_{i, j^{*}}^{k q}=\overline{\bar{N}}_{i, j^{*}}^{k}-\overline{\bar{N}}_{i, j^{*}}^{q} \\
\bar{b}_{j^{*}}^{k q}=\bar{b}_{j^{*}}^{k}-\bar{b}_{j^{*}}^{q}
\end{array}\right.
$$

$\bar{N}_{i, j^{*}}^{k q}$ is the intra-system single-difference wide-lane ambiguity between satellites $k$ and $q ; \overline{\bar{N}}_{i, j^{*}}^{k q}$ denotes the integer part of $\bar{N}_{i, j^{*}}^{k q}$, or nominally the integer wide-lane ambiguity, which has absorbed the integer part of wide-lane phase biases; $\bar{b}_{j^{*}}^{k q}$ represents the fractional part of wide-lane phase bias. Since only the fractional parts of phase biases matter in the recovery of integer single-receiver ambiguities, we simply take the fractional parts as the phase bias corrections throughout.

From Eq. 6, we can therefore compute the satellite-pair wide-lane phase biases through integer rounding operations, that is

$$
\bar{b}_{j^{*}}^{k q}=\left[\bar{N}_{i, j^{*}}^{k q}\right]-\bar{N}_{i, j^{*}}^{k q}
$$

where $[\cdot]$ is the integer rounding operator. The final phase bias corrections are usually estimated by averaging all obtainable $\bar{b}_{j^{*}}^{k q}$ from a reference network (Ge et al. 2008). Note that the phase bias products of the ultimate precision should be derived from a network solution enabling ambiguity-fixed clocks, though the sub- 
optimal phase biases calculated by the averaging operation can perform comparably well in positioning accuracy in case of short observation periods (Geng et al., 2019c; Teunissen and Khodabandeh, 2015).

However, $\bar{b}_{j^{*}}^{k q}$ is dedicated exclusively to the wide-lane ambiguities formed between frequencies $j$ and “*”, which would be junked by PPP users if carrierphase data on either frequency are not collected at their equipment. This situation will become even catastrophic if the data on the reference frequency "** are not collected by PPP-WAR users, because they would not be able to form wide-lane ambiguities with respect to frequency " *" to agree with the frequency combination prescribed by the wide-lane phase bias products $\bar{b}_{j^{*}}^{k q}$. One solution is to provide phase biases for all possible wide-lane frequency combination, which is however rather cumbersome and verbose in practice (e.g., $n$ frequencies means $C_{n}^{2}$ combinations). To mitigate this problem, at the server end we convert the wide-lane phase biases into frequencyspecific values by assigning a zero phase bias to the reference frequency. In particular,

$$
\left\{\begin{array}{l}
\bar{b}_{j}^{k q}=\bar{b}_{j^{*}}^{k q}+\bar{b}_{*}^{k q} \\
\bar{b}_{*}^{k q}=0
\end{array}\right.
$$

In this case, PPP-WAR users can still employ the phase bias specific to frequency $j$ even though data on frequency "**" are not collected or missing, rather than give up when delivered the frequency-pair phase bias products traditionally.

Once obtaining frequency-specific wide-lane phase biases, we can further decompose satellite-pair phase biases into satellite-specific or undifferenced (pseudo-absolute) values. For frequency $j$, that is

$$
\left\{\begin{array}{l}
\bar{b}_{j}^{k}=\bar{b}_{j}^{k r}+\bar{b}_{j}^{r} \\
\bar{b}_{j}^{q}=\bar{b}_{j}^{q r}+\bar{b}_{j}^{r} \\
\bar{b}_{j}^{r}=0
\end{array}\right.
$$


where $\bar{b}_{j}^{r}$ is the phase bias of the reference satellite $r$ which was assumed zero. In PPP-WAR, we will use these satellite- and frequency-specific phase biases to correct directly raw carrier-phase data in order to recover the integer property of singlereceiver ambiguities. It is worth stressing that these satellite- and frequency-specific phase biases are more flexible than the original satellite-pair and frequency-pair counterparts expressed in Eq. 7 in enabling wide-lane ambiguity fixing, since PPPWAR users are not limited to the wide-lane combinations corresponding to the delivered phase products $\bar{b}_{j^{*}}^{k q}$.

\subsection{Multi-frequency PPP-WAR}

The phase biases above can enable wide-lane ambiguity resolution at a single station. Different from Geng et al. (2019a) where PPP-WAR was only carried out using triplefrequency data, we integrated Galileo/BeiDou-3 data from all available frequencies to achieve PPP-WAR. Commencing again from the PPP solutions based on Eq. 2, we first used the phase bias products above to correct raw carrier-phase observations, which can be expressed as

$$
\left\{\begin{array}{l}
L_{i, 1}^{k}+\lambda_{s, 1} \bar{b}_{1}^{k}=\rho_{i}^{k}-\bar{\gamma}_{i}^{k}+\bar{t}_{i}^{s}-\bar{t}^{k}+\lambda_{s, 1} \tilde{N}_{i, 1}^{k} \\
L_{i, 2}^{k}+\lambda_{s, 2} \bar{b}_{2}^{k}=\rho_{i}^{k}-g_{s, 2}^{2} \bar{\gamma}_{i}^{k}+\bar{t}_{i}^{s}-\bar{t}^{k}+\lambda_{s, 2} \tilde{N}_{i, 2}^{k} \\
L_{i, j}^{k}+\lambda_{s, j} \bar{b}_{j}^{k}=\rho_{i}^{k}-g_{s, j}^{2} \bar{\gamma}_{i}^{k}+\bar{t}_{i}^{s}-\bar{t}^{k}+\lambda_{s, j} \tilde{N}_{i, j}^{k}
\end{array}\right.
$$

and then the raw ambiguities become theoretically

$$
\left\{\begin{array}{l}
\tilde{N}_{i, 1}^{k}=\bar{N}_{i, 1}^{k}+\bar{b}_{1}^{k} \\
\tilde{N}_{i, 2}^{k}=\bar{N}_{i, 2}^{k}+\bar{b}_{2}^{k} \\
\tilde{N}_{i, j}^{k}=\bar{N}_{i, j}^{k}+\bar{b}_{j}^{k}
\end{array}\right.
$$

where $\tilde{N}_{i, 1}^{k}, \tilde{N}_{i, 2}^{k}$ and $\tilde{N}_{i, j}^{k}$ denote undifference ambiguities which are now free from the contamination of fractional satellite phase biases. We further form intrasystem single-difference ambiguity estimates to eliminate receiver-specific phase biases and finally obtain integer single-difference ambiguities. These single-difference 
ambiguities together with their variance-covariance matrix are further converted into their wide-lane counterparts with respect to a reference frequency (cf. Dong and Bock, 1989). To be specific, in case of five-frequency data, we have

$$
\left(\begin{array}{c}
\overline{\bar{N}}_{i, 12}^{k q} \\
\check{\bar{N}}_{i, 13}^{k q} \\
\check{\bar{N}}_{i, 14}^{k q} \\
\overline{\bar{N}}_{i, 15}^{k q}
\end{array}\right)=\left(\begin{array}{ccccc}
1 & -1 & 0 & 0 & 0 \\
1 & 0 & -1 & 0 & 0 \\
1 & 0 & 0 & -1 & 0 \\
1 & 0 & 0 & 0 & -1
\end{array}\right)\left(\begin{array}{c}
\tilde{N}_{i, 1}^{k q} \\
\tilde{N}_{i, 2}^{k q} \\
\tilde{N}_{i, 3}^{k q} \\
\tilde{N}_{i, 4}^{k q} \\
\tilde{N}_{i, 5}^{k q}
\end{array}\right)
$$

where $\stackrel{\bar{N}}{N}_{i, 1 j}^{k q}(j=2,3,4,5)$ denotes the wide-lane ambiguities to be resolved; frequency " 1 " is considered the reference frequency. The mapping matrix in Eq. 12 is also needed to convert the original variance-covariance matrix into that for wide-lane ambiguities (cf. Geng et al., 2012). We note that, though Eq. 12 is derived from a fivefrequency functional model, it can actually be extended to any number of frequencies by expanding the mapping matrix. We then inject all $\overline{\bar{N}}_{i, 1 j}^{k q}$ and their variancecovariance matrix into the LAMBDA (Least-squares Ambiguity Decorrelation Adjustment) method simultaneously to search for their integer candidates (Teunissen 1995). At least two wide-lane ambiguities have to be resolved successfully for each satellite before we accomplish PPP-WAR. Unless when otherwise noted, we carry out PPP-WAR using only a single epoch of data, which means that both ambiguity and atmosphere parameters are computed without any sort of multi-epoch filtering. In this case, instant PPP-WAR has the highest potential of recovering instantaneously from signal blockages, or the highest capability of resisting carrier-phase discontinuities. In addition, we inject all resolvable wide-lane ambiguities into the integer least-squares estimator simultaneously, which differs from Geng and Bock (2013) where extrawide-lane and wide-lane ambiguities were fixed to integers sequentially.

\subsection{Remarks on the extendibility and flexibility of multi-frequency PPP-WAR}

Sections 2.1 and 2.2 describes the algorithms used to compute phase biases at the 
server end and to perform PPP-WAR at the user end. We demonstrate that the extendibility and flexibility of this approach when applied to multi-frequency and multi-constellation GNSS data lies in three aspects. At first, uncombined GNSS model is employed to process GNSS data as shown in Eq. 2. We can thus easily introduce as many multi-frequency and multi-GNSS data as possible if inter-system code biases and inter-frequency clock biases are considered properly (cf. Guo and Geng, 2017). Second, we derive satellite- and frequency-specific phase biases using Eqs. 8 and 9, instead of their satellite-pair and frequency-pair counterparts initially estimated with Eq. 7. In this manner, we do not need to provide cumbersome widelane phase biases corresponding to all possible frequency-pair combinations, whose number grows nearly exponentially with the increasing number of frequencies. Finally, PPP-WAR users directly correct their raw carrier-phase observations for satellite- and frequency-specific phase biases without resorting to any explicit formations of any particular wide-lane combination observables, which is also a desired manner suggested by the IGS bias-SINEX (Software INdependent EXchange) conventions (Schaer 2016). Though wide-lane ambiguities are formulated and variance-covariance matrix are converged later at the normal equation level, this derivation is to make sure that no narrow-lane ambiguities should be resolved automatically in the integer least-squares estimator. It is also worth stressing that it does not matter which wide-lane combinations are formulated at the normal equation level, as long as $n-1$ of them are constituted where $n$ is the number of frequencies involved.

\section{Data processing}




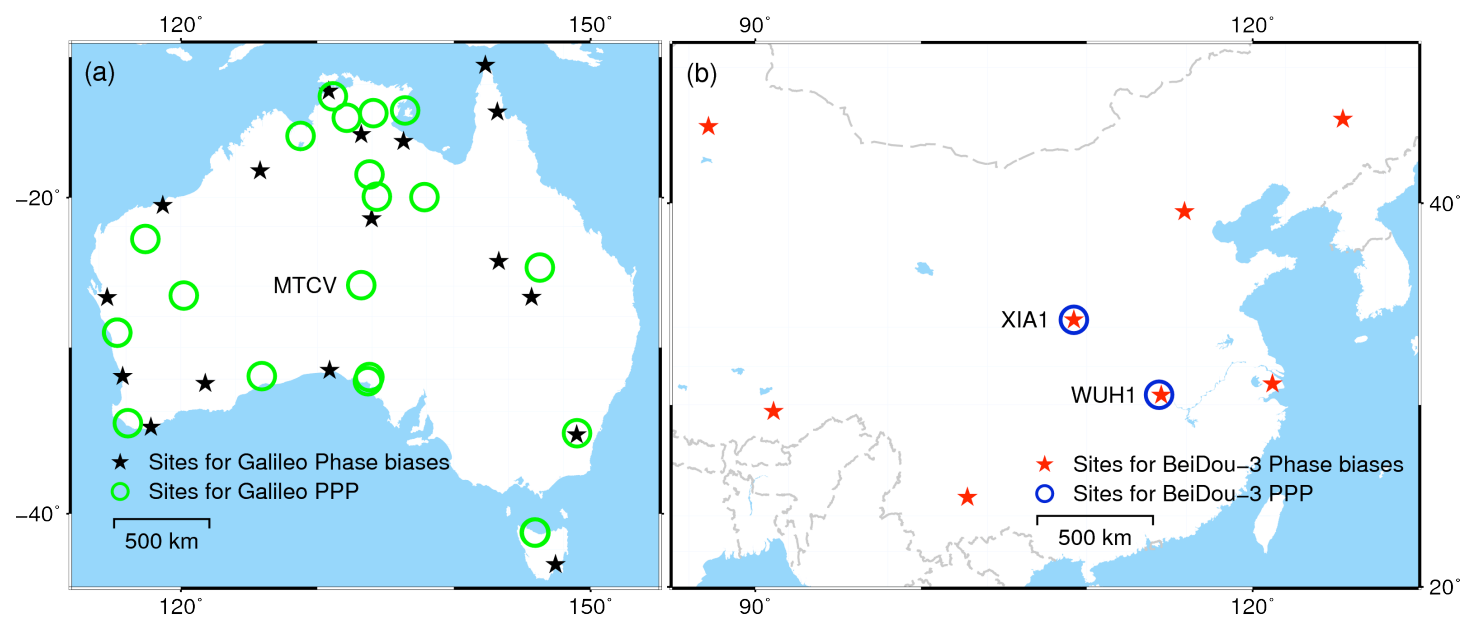

Figure 1 Distribution of Galileo stations from the Australian Regional GNSS Network (ARGN) (panel a), and BeiDou-3 stations from the International GNSS Monitoring \& Assessment System (IGMAS) (panel b). Stations denoted as black and red solid stars are used to estimate phase bias products for Galileo and BeiDou-3, respectively, while those denoted as green and blue open circles contribute to Galileo and BeiDou-3 PPP-WAR tests. Three stations MTCV, XIA1 and WUH1 are especially marked for further reference in the main text.

To analyze the performance of Galileo/BeiDou-3 multi-frequency instant PPP-WAR, 30-s data spanning days 326-356 in 2018 at 36 stations from the Australian Regional GNSS Network (ARGN) were collected (Fig. 1a); 19 of them were selected for Galileo PPP-WAR solutions while the other 17 for Galileo clock/phase bias estimation. It is worth mentioning that in ARGN only Septentrio receivers could receive Galileo E6 signals and hence all 36 stations chosen for this study employed SEPT PolaRx5 receivers (Table 2). Since multi-frequency BeiDou-3 signals could not be received by ARGN, we also obtained seven days (days 035-041 in 2019) of the International GNSS Monitoring \& Assessment System (IGMAS) data from 10 stations distributed across China for BeiDou-3 clock/phase bias estimation and PPPWAR validations (Table 2) (Fig. 1b). The final multi-GNSS orbit products computed by the Center for Orbit Determination in Europe (CODE) were used for Galileo, whereas those from Wuhan University were used for BeiDou-3 (Zhao et al., 2017); in this case, both Galileo and BeiDou-3 satellite clocks were re-estimated. Note that we used only Galileo E1/E5a and BeiDou-3 B1C/B2a dual-frequency data to compute 
satellite clocks, but applied them to multi-frequency PPP-WAR by presuming the absence of time-varying inter-frequency clock biases (Zhang et al., 2017; Geng et al., 2019a). Zenith troposphere delays (ZTDs) were mitigated a priori using the Saastamoinen model and then estimated hourly using the global mapping function alongside a process noise of $2 \mathrm{~cm} / \sqrt{h}$ (Boehm et al. 2006; Saastamoinen 1973). We did not apply any differential code bias (DCB) corrections to Galileo data since all 36 ARGN receivers had favorably the same types of pseudorange observables (Table 2). However, this was not the case of the 10 IGMAS stations; unfortunately we were short of BeiDou-3 DCBs. Consequently, BeiDou-3 DCBs were recklessly presumed zero throughout. In addition, the igs14_2032.atx file was used to calibrate the satellite and station antenna phase center offset/variations (PCO/PCVs) for Galileo and BeiDou-3. Of particular note, regarding station PCO/PCVs, we simply used the GPS L1 corrections to approximate those on B1/E1, while all other frequencies shared the same station PCO/PCVs as those of GPS L2. Note that the PCO/PCVs for BeiDou-3 satellites here were the same as those applied to the orbit determination by Zhao et al. (2017). In addition, Galileo wide-lane phase biases were estimated hourly while BeiDou-3 phase biases every 15 minutes. BeiDou-3 phase biases were estimated more often because the loss of BeiDou-3 signals at the IGMAS stations took place frequently within an hour.

Table 2 Receivers, antennas and pseudorange observables at the 36 ARGN stations and 10 IGMAS stations according to IGS RINEX (Receiver INdependent Exchange) ver. 3.04. One of the 10 IGMAS station was actually a self-established temporary station employing a Trimble Alloy receiver located in Wuhan University. The last column shows the number of stations equipped with such types of receivers and antennas.

\begin{tabular}{llll}
\hline Receivers & Pseudorange & Antennas & Number \\
\hline ARGN stations for & Galileo PPP-WAR & & \\
SEPT PolaRx5 & Galileo: & JAVRINGANT_DM & 6 \\
\hline
\end{tabular}




\begin{tabular}{lll}
\hline C1C/C5Q/C6C/C7Q/C8Q & LEIAR25.R3 & 14 \\
\cline { 2 - 3 } & LEIAR25.R4 & 1 \\
\cline { 2 - 3 } LEIAR25 & 1 \\
\cline { 2 - 3 } LEIAT504 & 3 \\
\hline LEIAT504GG & 3 \\
\hline AOAD/M_T & 3 \\
\hline TRM59800.00 & 4 \\
\hline & ASH701945C_M & 1 \\
\hline
\end{tabular}

IGMAS stations for BeiDou-3 PPP-WAR

\begin{tabular}{llll} 
GMR-4016 & BeiDou-3: & LEIAR25.R4 & 2 \\
\cline { 3 - 4 } & C1X/C2I/C5X/C6I/C7I & NOV750.R4 & 2 \\
\hline GNSS-GGR & BeiDou-3: & RINT-8CH & 3 \\
& C1X/C2I/C5X/C6I/C7I & & 2 \\
\hline UB4B0-13478 & BeiDou-3: & NOV750.R4 & 2 \\
& C1X/C2I/C5X/C6I & & \multirow{2}{*}{1} \\
Trimble Alloy & BeiDou-3: & TRM115000.00 & \\
(Self-established & $\mathrm{C} 1 \mathrm{X} / \mathrm{C} 2 \mathrm{I} / \mathrm{C} 5 \mathrm{X} / \mathrm{C} 6 \mathrm{I}$ & & \\
station $)$ & & & \\
\hline
\end{tabular}

Vehicle-borne experiment

Trimble Alloy Galileo: HG-GOYH7151 1

C1X/C5X/C6X/C7X/C8X

BeiDou-3:

C1X/C2I/C5X/C6I

Table 3 Data processing strategies and models for Galileo/BeiDou-3 multi-frequency data.

\begin{tabular}{ll}
\hline Items & Strategies \\
\hline Frequencies & Galileo: E1/E5a/E5/E5b/E6; \\
& BeiDou-3: B1C/B1I/B2a/B2b/B3I.
\end{tabular}


Cut-off angle

Weighting

Positions

Receiver clocks

Inter-system code bias

Inter-frequency clock bias

Ionosphere delays

Troposphere delays

Ambiguities $10^{\circ}$

Elevation-dependent;

3-mm and 0.3-m a priori noise for carrier-phase

and pseudorange, respectively.

White-noise like parameters.

White-noise like parameters.

Constant per day.

Constant per day.

Random-walk parameter with a process noise of

$25 m / \sqrt{30 s}$.

Not estimated but only a priori corrected with the

Saastamoinen model.

White-noise like parameters.

For instant PPP-WAR, we estimated positions, receiver clocks, slant ionosphere delays and ambiguities epoch by epoch by imposing quite weak temporal constraints on neighboring epochs (Table 3), except the inter-system code and inter-frequency clock biases as constants over a day. ZTDs were not estimated though. For the comparison on instant positioning, we also ran "single-epoch PPP" whose only difference from instant PPP-WAR was that no ambiguities were resolved; in fact, single-epoch PPP was the same as standard point positioning except for the precise satellite corrections and sophisticated PPP models. Furthermore, we searched for the integer candidates simultaneously for all wide-lane ambiguities through LAMBDA, and a threshold of 3.0 was set for the ratio test to distinguish between the optimal and second optimal integer candidates (Euler and Schaffrin, 1990). If the ratio test failed, we would switch to partial ambiguity fixing where we eliminated up to four ambiguities while keeping at least four for a second ambiguity-fixing trial; if the ratio threshold could not be satisfied finally, a float solution was kept eventually. 


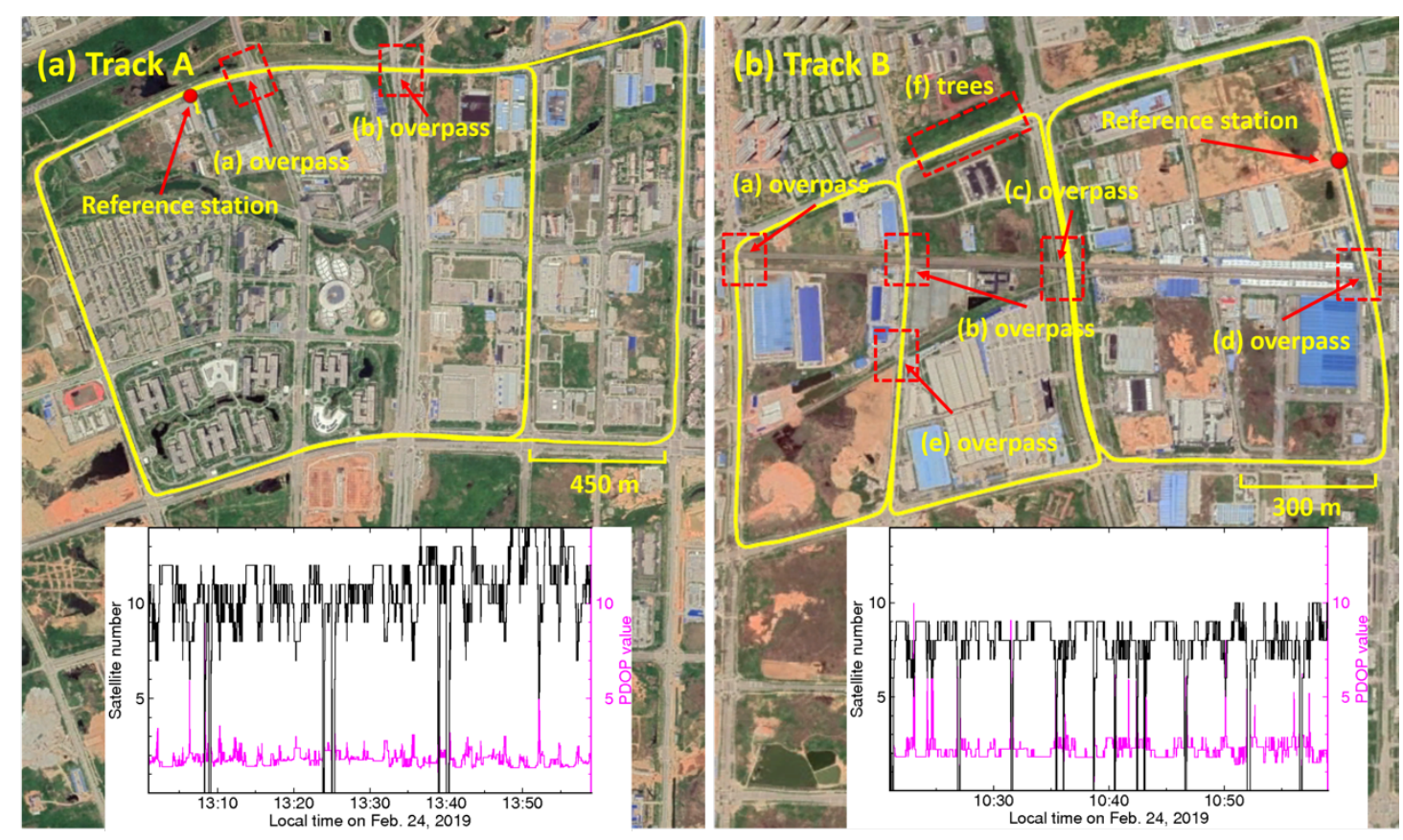

Figure 2 Vehicle trajectories (yellow lines) along tracks A and B on February 24, 2019 overlaid on Google maps. Eight dashed red squares along the routes denote seven overpasses and one passage seriously shaded by trees. The two red solid circles represent reference stations established for short-baseline benchmark solutions. The two insets show the number of visible Galileo/BeiDou-3 satellites (black lines) and position dilution of precision (PDOP) (purple lines) during the vehicleborne experiments.

We also carried out two vehicle-borne experiments on February 24, 2019 in Wuhan city, China (near WUH1 in Fig. 1b). The vehicle drove three laps at a speed of about $30 \mathrm{~km} / \mathrm{h}$ along tracks $\mathrm{A}$ and $\mathrm{B}$, totaling about $8.4 \mathrm{~km}$ and $8 \mathrm{~km}$, respectively. Specifically, from 13:00 until 14:00 local time, we drove along Track A when about 57 BeiDou-3 satellites could be observed; we also drove along Track B from 10:20 until 11:00 local time. A latest Trimble Alloy receiver which was able to track Galileo E1/E5a/E5/E5b/E6 and BeiDou-3 B1C/B1I/B2a/B3I signals was placed within the vehicle while connected with a HG-GOYH7151 antenna on top of the vehicle. The data processing strategy for the Alloy receiver data was the same as that for the PPPWAR stations in Fig. 1 (Table 2). It is worth mentioning that pseudorange data on B1I from Trimble Alloy suffered from abnormal periodic jumps, and neither B1I pseudorange nor carrier-phase were usable in this study. Moreover, a Trimble NetR9 
receiver connected to a Zephyr Geodetic 2 antenna, collecting 1-Hz Galileo/BeiDou-3 data, was used as the reference station and placed near each experiment area (see Fig.

2) to provide short-baseline benchmark solutions. Note that for vehicle-borne

Galileo/BeiDou-3 PPP-WAR, all stations in Fig. 1 were still used for the phase bias estimation.

\section{Results}

\subsection{Undifferenced uncombined phase biases}

(a) $\bullet \mathrm{E} 01 \bullet \mathrm{E} 02 \cdot \mathrm{E} 03 \cdot \mathrm{E} 04 \bullet \mathrm{E} 05 \bullet \mathrm{E} 07 \bullet \mathrm{E} 08 \bullet \mathrm{E} 09 \bullet \mathrm{E} 11 \bullet \mathrm{E} 12 \bullet \mathrm{E} 13 \bullet \mathrm{E} 14 \bullet \mathrm{E} 15$

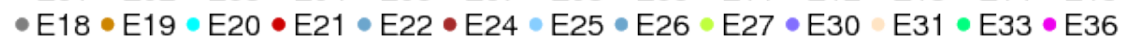

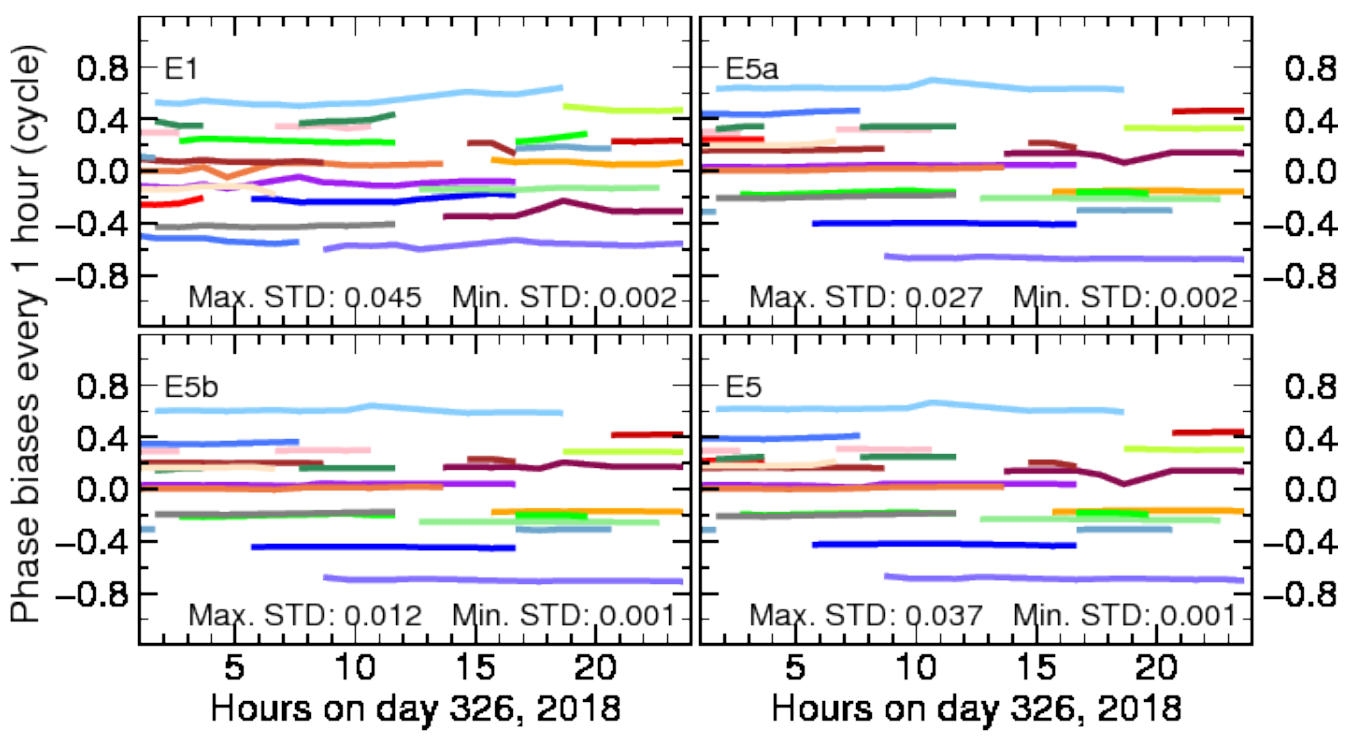

(b) $\quad \mathrm{C} 19 \bullet \mathrm{C} 20 \bullet \mathrm{C} 21 \bullet \mathrm{C} 22 \bullet \mathrm{C} 23 \bullet \mathrm{C} 24 \cdot \mathrm{C} 25 \bullet \mathrm{C} 26 \bullet \mathrm{C} 27 \bullet \mathrm{C} 28 \bullet \mathrm{C} 29 \bullet \mathrm{C} 30 \bullet \mathrm{C} 31$

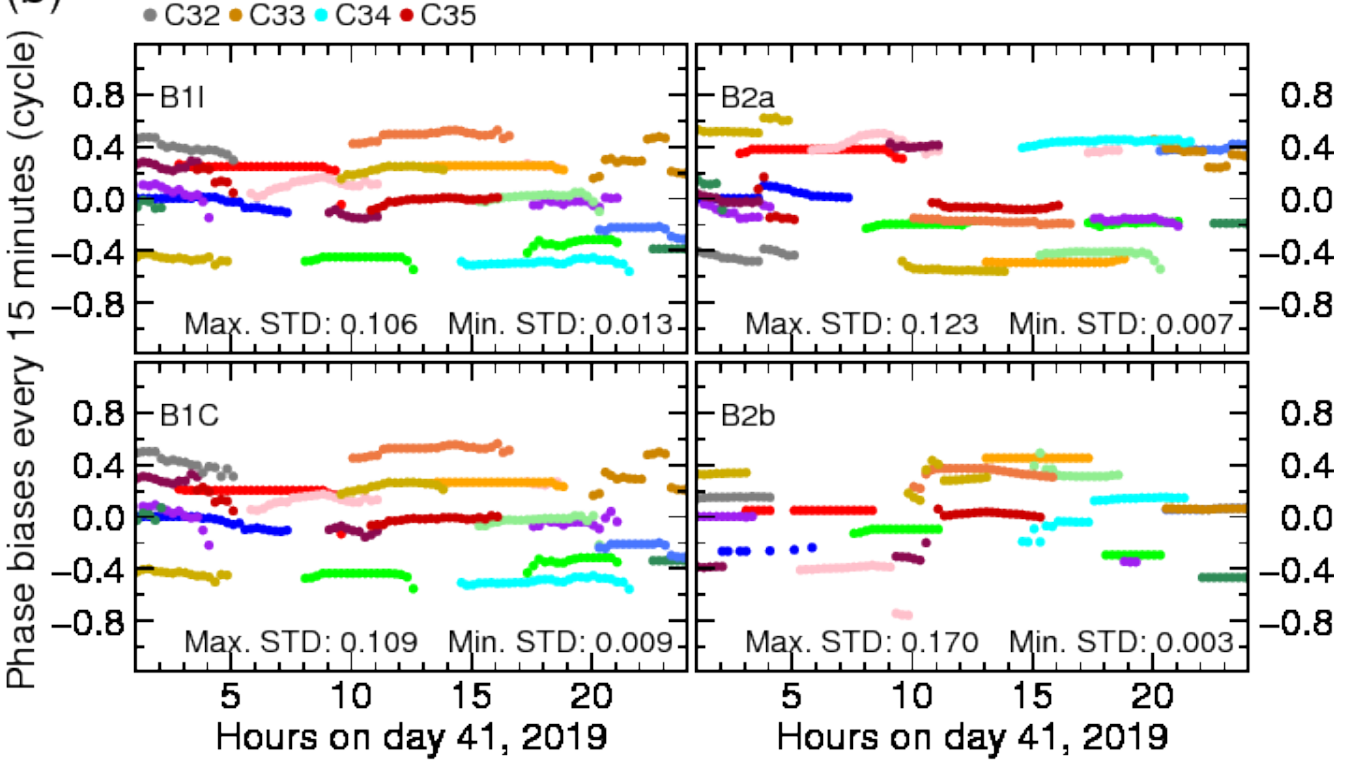

Figure 3 Typical satellite- and frequency-specific Galileo E1/E5a/E5/E5b (panel a) and BeiDou-3 
B1I/B1C/ B2a/B2b (panel b) wide-lane phase biases estimated every hour on day 326 of 2018 and every 15 minutes on day 041 of 2019 , respectively. Note that all phase bias traces have been offset deliberately to avoid overlap of symbols. "STD" stands for "standard deviation".

After PPP processing at all reference stations in Fig. 1, we can form single-difference wide-lane ambiguity estimates (Eqs. 4 and 5), whose fractional parts are identified by Eq. 7 and finally converted to satellite- and frequency-specific phase biases through Eqs. 8 and 9. Fig. 3 show the typical time series of multi-frequency phase bias estimates for Galileo and BeiDou-3 on the days when a large number of satellites were visible to the ARGN and IGMAS stations; the phase biases for all available frequencies are shown except for the reference E6 and B3I signals.

Table 4 The mean standard deviations (cycles) of wide-lane phase biases for Galileo and BeiDou-3 satellites spanning days 326-355, 2018 and days 35-41, 2019, respectively.

\begin{tabular}{lllll}
\hline GNSS & E1/B1I & E5a/B3I & E5b/B1C & E5/B2b \\
\hline Galileo & 0.016 & 0.006 & 0.004 & 0.006 \\
BeiDou-3 & 0.030 & 0.029 & 0.036 & 0.033 \\
\hline
\end{tabular}

Overall, the hourly Galileo phase bias time series show an excellent temporal stability whose standard deviations are all less than 0.05 cycles, evidenced by the almost perfectly flat traces for all satellites plotted in Fig. 3a; moreover, the mean standard deviation over all 30 days is below 0.02 cycles. However, we found that the standard deviations of E1 phase biases are roughly three times larger than those of $\mathrm{E} 5 \mathrm{a} / \mathrm{E} 5 / \mathrm{E} 5 \mathrm{~b}$ (Table 4). This phenomenon can be understood in terms of the almost three times longer wavelengths of the wide-lane ambiguities formed between E5a/E5/E5b and E6 in contrast to that between E1 and E6. Moreover, when applying such Galileo phase bias corrections to the wide-lane ambiguities at the reference stations, the residual fractional parts of single-difference ambiguities are rather small 
with a mean RMS of 0.01 cycles, suggesting the high precisions of Galileo phase bias estimates.

On the contrary, applying BeiDou-3 phase bias corrections to the stations in Fig. $1 \mathrm{~b}$ results in a mean RMS of ambiguity fractional parts of about 0.05 cycles, implying an inferior precision for BeiDou-3 phase biases in this study. At first glance, the 15min BeiDou-3 phase bias time series in Fig. 3b appear to be smooth generally with the minimum standard deviation of below 0.01 cycles. However, there are still a number of large fluctuations of up to 0.1 cycles lasting several hours, and pronounced jumps of up to 0.2 cycles present in the BeiDou- 3 phase bias traces, ultimately contributing to the maximum standard deviations of over 0.1 cycles plotted at the bottom of each panel of Fig. 3b. On average, the BeiDou-3 phase bias standard deviation for each relevant frequency is about 0.03 cycles over the seven days, roughly three times worse than those of Galileo (Table 4). We find that the significant fluctuations and clear discontinuities within BeiDou-3 phase biases in Fig. $3 \mathrm{~b}$ are caused in the main by the limited number of the reference stations observing BeiDou-3 satellites simultaneously (i.e., we have only 10 IGMAS stations in total). Of particular note, the stations equipped with local receiver types often malfunctioned when tracking BeiDou-3 signals, resulting in a large number of cycle slips and data gaps (i.e., receivers GMR4016, GNSS-GGR and UB4B0-13478 in Table 2). Such deficiency in station redundancy deteriorates the precision and reliability of averaging the fractional parts of ambiguities over all IGMAS stations. This also explains why we computed BeiDou-3 phase biases more often to avoid even longer discontinuities.

\subsection{Multi-frequency instant PPP-WAR using ARGN and IGMAS stations}




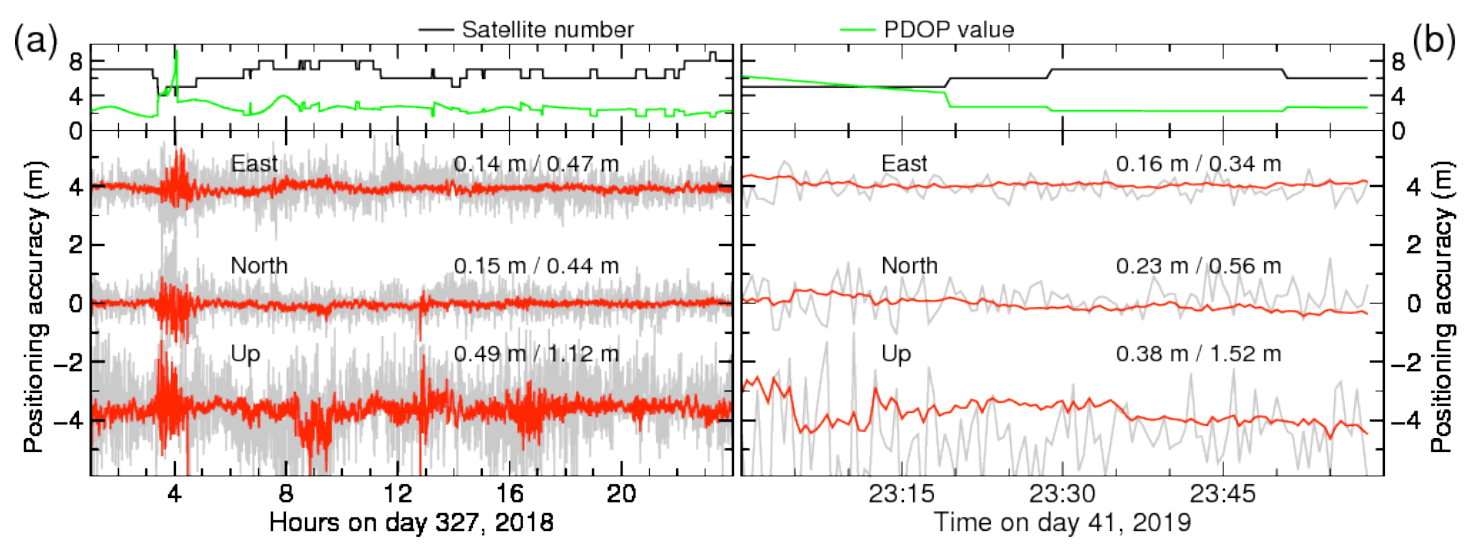

Figure 4 Positioning accuracy (m) of Galileo-only (panel a for station MTCV) and BeiDou-3 only (panel $b$ for station XIA1) five-frequency instant PPP-WAR (red traces) and single-epoch PPP (gray traces). For symbol clarity, the east and up components are offset by 4 and $-4 \mathrm{~m}$, respectively. The RMS statistics are shown alongside each trace where the numerals before "/" are for five-frequency instant PPP-WAR and those after are for single-epoch PPP. Satellite numbers and PDOP values are plotted inside the top panels. "Single-epoch PPP" throughout means a float PPP solution using only a single epoch of data.

With multi-frequency phase bias products, we are able to implement instant PPPWAR on Galileo and BeiDou-3 data from the stations in Fig. 1. At first, Galileo-only and BeiDou-3 only PPP-WAR were both carried out based on the five-frequency $\mathrm{E} 1 / \mathrm{E} 5 \mathrm{a} / \mathrm{E} 5 / \mathrm{E} 5 \mathrm{~b} / \mathrm{E} 6$ and B1C/B1I/B2a/B2b/B3I data. The positioning accuracy was quantified by taking daily position estimates as the truth benchmark. Fig. 4a shows the positioning accuracy of both Galileo-only five-frequency instant PPP-WAR (red traces) and single-epoch PPP (gray traces) solutions for station MTCV (cf. Fig. 1) on day 327, 2018. Compared to single-epoch PPP, five-frequency instant PPP-WAR improves dramatically the positioning accuracy for the east, north and up components from $0.47,0.44,1.12 \mathrm{~m}$ to $0.14,0.15$ and $0.49 \mathrm{~m}$, respectively, showing a $70 \%$ reduction in terms of RMS statistics. Table 5 summarizes the average performance for all ARGN Galileo stations, confirming the achievement revealed at station MTCV. Moreover, five-frequency instant PPP-WAR solutions using BeiDou-3 data only are presented for station XIA1 in Fig. 4b; here we only show one hour of results when at least 5-6 BeiDou-3 satellites were visible. An RMS of 0.16, 0.23 and $0.38 \mathrm{~m}$ is 
achieved for the east, north and up components, respectively, in contrast to $0.34,0.56$ and $1.52 \mathrm{~m}$ for single-epoch BeiDou-3 PPP; this fact demonstrates the comparable potential of BeiDou-3 to that of Galileo for instant decimeter-level positioning.

Table 5 The mean RMS (m) of the position differences for both instant five-frequency PPP-WAR and single-epoch PPP solutions from daily position benchmarks in case of different Galileo satellite numbers at all 36 ARGN stations over the 30 days. The RMS are shown from the east, north to up components delimited by “/”. Column 4 shows the reduction rate (\%) of the horizontal RMS when comparing instant PPPWAR to single-epoch PPP. Column 5 shows the percentages of epoch-wise solutions whose position differences from benchmarks fall in $\pm 0.3 \mathrm{~m}$ for both east and north components; the numerals before "/" denote the percentages for PPP-WAR while those after for single-epoch PPP.

\begin{tabular}{lllll}
\hline $\begin{array}{l}\text { Satellite } \\
\text { number }\end{array}$ & $\begin{array}{l}\text { Five-freq. PPP- } \\
\text { WAR }\end{array}$ & $\begin{array}{l}\text { Single-epoch } \\
\text { PPP }\end{array}$ & $\begin{array}{l}\text { Reduction } \\
\text { rate }\end{array}$ & $\begin{array}{l}\text { Within } \pm \mathbf{0 . 3} \\
\text { m }\end{array}$ \\
\hline $5-6$ & $0.21 / 0.20 / 0.66$ & $0.50 / 0.49 / 1.18$ & $58.6 \%$ & $82.0 \% / 32.3 \%$ \\
$7-8$ & $0.16 / 0.14 / 0.58$ & $0.42 / 0.40 / 1.05$ & $63.7 \%$ & $90.3 \% / 37.9 \%$ \\
$9-10$ & $0.10 / 0.11 / 0.48$ & $0.31 / 0.30 / 0.85$ & $65.9 \%$ & $93.8 \% / 42.5 \%$
\end{tabular}

Table 6 The mean RMS (m) of the position differences for Galileo-only instant fivefrequency and triple-frequency PPP-WAR from daily position benchmarks at the 36 ARGN stations over the 30 days. The RMS are shown from the east, north to up components delimited by “/”. Column 3 shows the success rate of PPP-WAR solutions within all epochs.

\begin{tabular}{llll}
\hline Model & PPP-WAR & Success rate & Single-epoch PPP \\
\hline Five-freq. PPP-WAR & $0.20 / 0.19 / 0.65$ & $99.5 \%$ & $0.49 / 0.48 / 1.19$ \\
using E1/E5a/E5/E5b/E6 & & & \\
Triple-freq. PPP-WAR & $0.20 / 0.18 / 0.65$ & $99.6 \%$ & $0.51 / 0.49 / 1.24$ \\
using E1/E5a/E6 & & & \\
Triple-freq. PPP-WAR & $0.31 / 0.29 / 0.87$ & $98.4 \%$ & $0.50 / 0.48 / 1.21$ \\
\hline
\end{tabular}


using $\mathrm{E} 1 / \mathrm{E} 5 \mathrm{a} / \mathrm{E} 5 \mathrm{~b}$

Triple-freq. PPP-WAR $\quad 0.35 / 0.33 / 0.93 \quad 97.6 \%$

$0.49 / 0.48 / 1.19$ using E1/E5a/E5

For comparison, we also tried instant triple-frequency PPP-WAR in case of different frequency combinations such as Galileo E1/E5a/E6, E1/E5a/E5b, and E1/E5a/E5. Table 6 thus contrasts the mean positioning RMS of both five-frequency and triple-frequency Galileo-only PPP-WAR solutions. Overall, wide-lane ambiguities are all fixed with success rates of over 97\%, while five-frequency PPP-WAR performs relatively better, though slightly. Interestingly, similar positioning RMS are achieved by five-frequency and triple-frequency PPP-WAR whenever the E6 signal is involved; if E6 is excluded from PPP-WAR, however, the horizontal positioning RMS will be degraded by about 50\% (cf. Laurichesse and Banville, 2018). Conversely, this benefit of improved positioning accuracy attributed to the E6 signals is not visible anymore if no ambiguities are resolved as evidenced by single-epoch PPP solutions in Table 6 .

Although similar positioning RMS are achieved whenever the E6 signals are involved in instant multi-frequency Galileo-only PPP-WAR, it does not undermine the necessity of using all available frequencies of data for instant decimeter-level positioning. We argue that the foremost advantage of using all available frequencies consists in the higher availability of precise positions, especially in response to any loss of signals on some frequencies. To experiment on this argument, we simulated the loss-of-track events by removing the raw carrier-phase observables for the first $n$ satellites at each epoch in the RINEX files. The losses of E6, E5b and E5 signals from 1-4 satellites were simulated for E1/E5a/E6, E1/E5a/E5b and E1/E5a/E5 PPP-WAR, respectively, while the loss of E6, E5b or E5 is presumed separately for fivefrequency PPP-WAR. Fig. 5 shows the results where the "availability of precise positioning" is defined as the percentage of epoch-wise positions whose east and north positioning differences from the benchmarks both fall within $\pm 0.3 \mathrm{~m}$. All 36 
ARGN stations from all days were counted. We can find that the availability of fivefrequency instant PPP-WAR (dashed lines) always exceed those of triple-frequency PPP-WAR (solid lines). In particular, the highest availability of up to $87 \%$ can be always achieved in case of five-frequency PPP-WAR as long as the E6 signals are not lost, no matter how many satellites suffer from the loss of E5b/E5 signals. Even though the E6 signals are not tracked for up to five satellites, an availability of $72 \%$ can still be kept for five-frequency PPP-WAR. In contrast, if the E6 signals of five satellites are lost for E1/E5a/E6-enabled PPP-WAR, these satellites will not be able to contribute to PPP-WAR solutions anymore, therefore reducing the precise positioning availability to only $51 \%$.

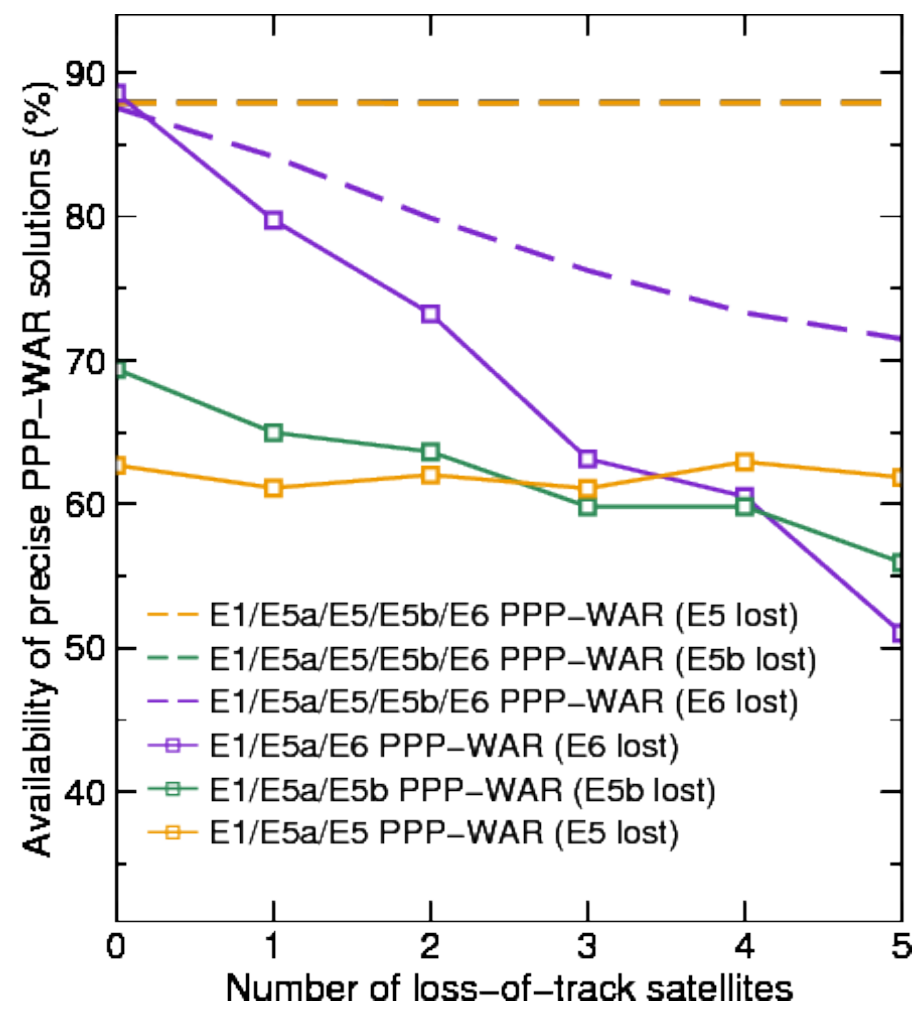

Figure 5 Availability of precise positioning for Galileo-only instant five-frequency and triplefrequency PPP-WAR solutions corresponding to those in Table 6, when some specific frequencies of carrier-phase signals are lost for different number of satellites. For example, "E1/E5a/E6 PPPWAR (E6 lost)" denotes triple-frequency PPP-WAR when the E6 signals are lost for 1-5 satellites. "Precise positioning" means that the positioning accuracy for the horizontal components falls in $\pm 0.3 \mathrm{~m}$. Note that the dashed purple and green lines overlap each other. 


\subsection{Vehicle-borne Galileo/BeiDou-3 instant PPP-WAR experiment}

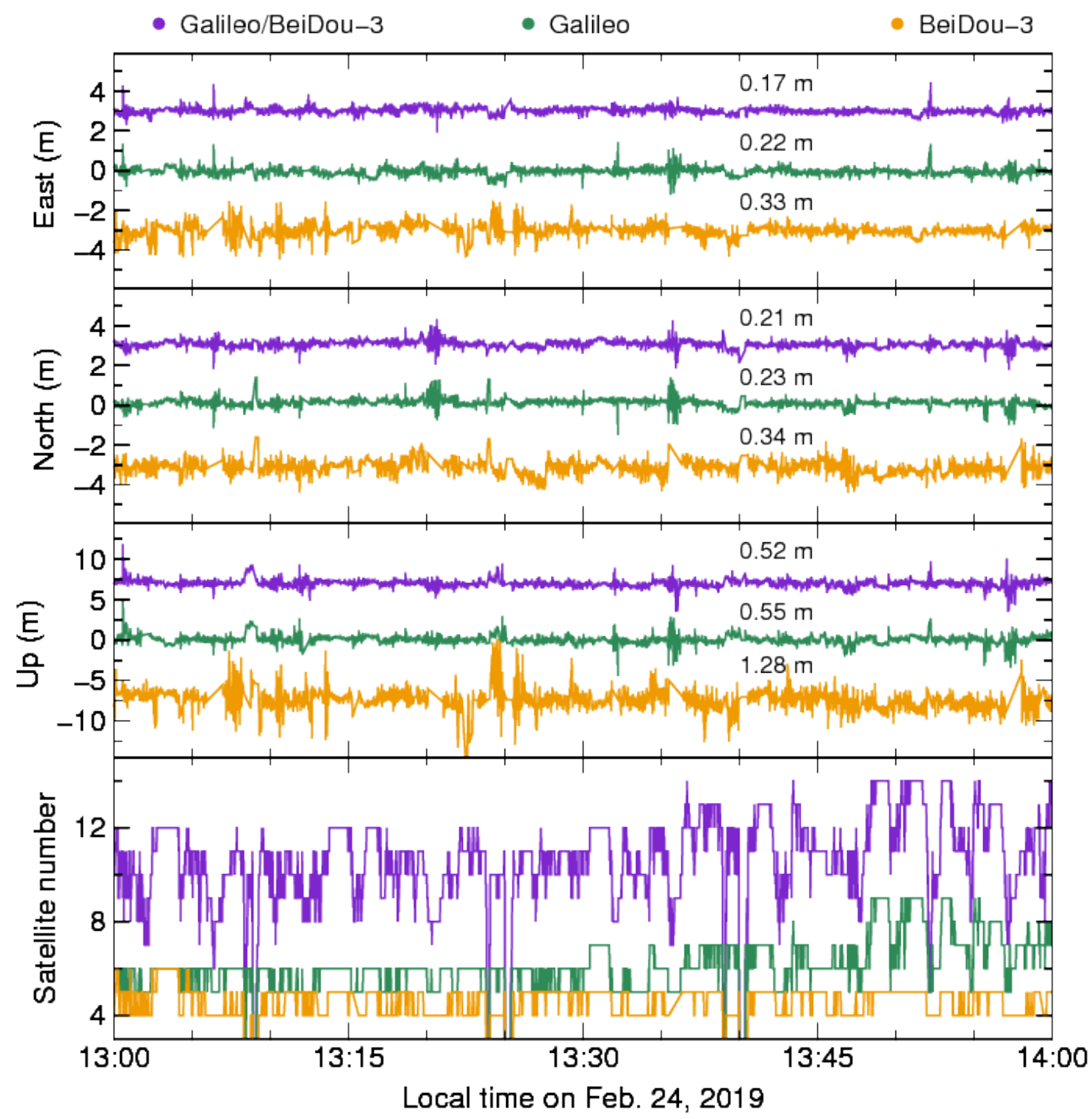

Figure 6 Positioning accuracy (m) for the east, north and up components of instant five-frequency PPP-WAR solutions for the track A experiment over a 1-hour duration on February 24, 2019.

Short-baseline solutions are taken as the truth benchmarks (cf. Fig. 2a). We tried Galileo/BeiDou3, Galileo-only and BeiDou-3 only PPP-WAR, whose RMS statistics are plotted alongside the traces of positioning differences. Note that the purple and orange traces have been offset by $3 \mathrm{~m}$ for the east and north components, while $7.5 \mathrm{~m}$ for the up component for clarity. The number of visible Galileo/BeiDou-3 satellites is displayed inside the bottom panel. 


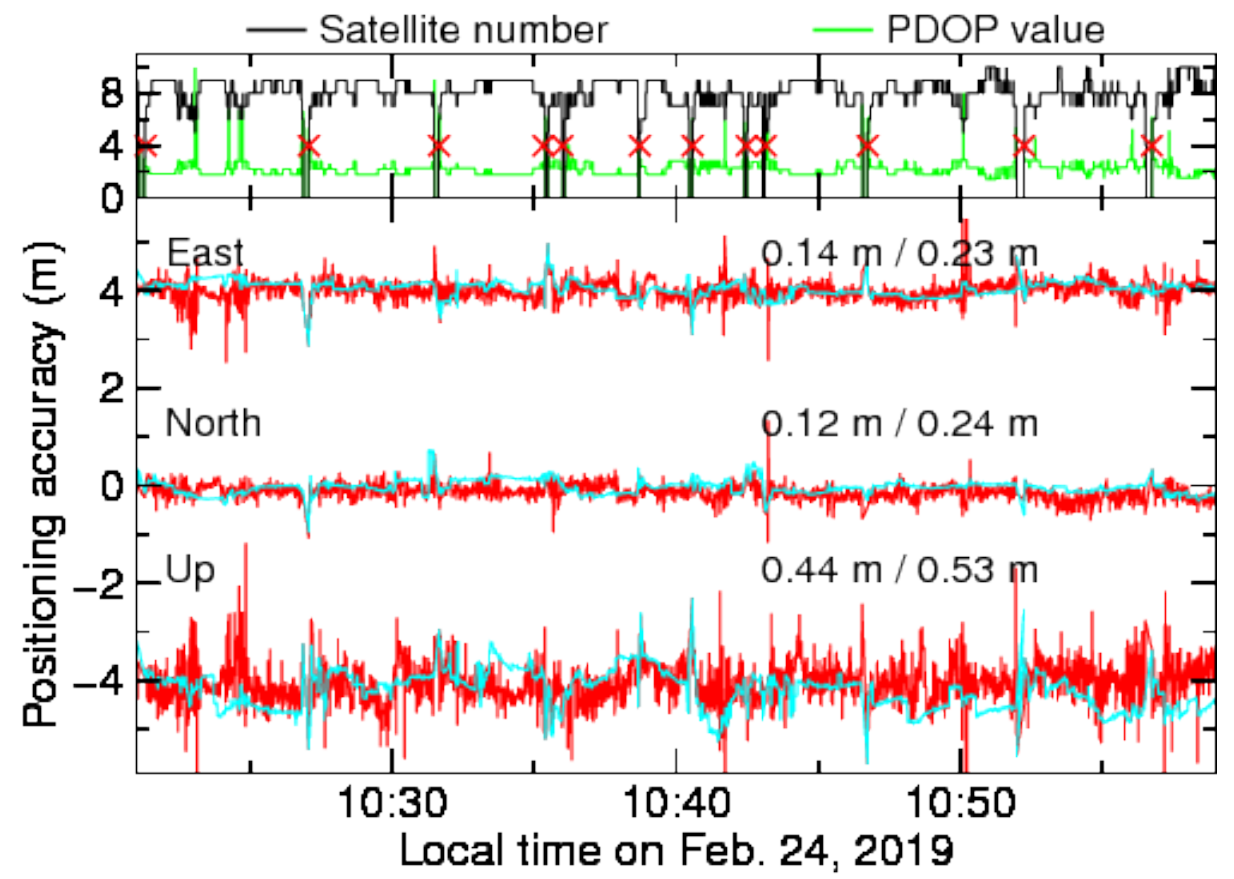

Figure 7 Positioning accuracy (m) of multi-frequency Galileo/BeiDou-3 instant PPP-WAR (red lines) and multi-epoch PPP-WAR (cyan lines) solutions for the vehicle driving on track B over a duration of 40 minutes. Short-baseline solutions are taken as truth benchmarks (cf. Fig. 2b). The east and up components of both solutions are offset by $4 \mathrm{~m}$ to avoid overlap of symbols. The top panel shows the number of visible satellites and PDOP values, which is the same as the inset in Fig. 2b. The 12 red crosses mark the total loss-of-track events caused by overpasses in Fig. 2b.

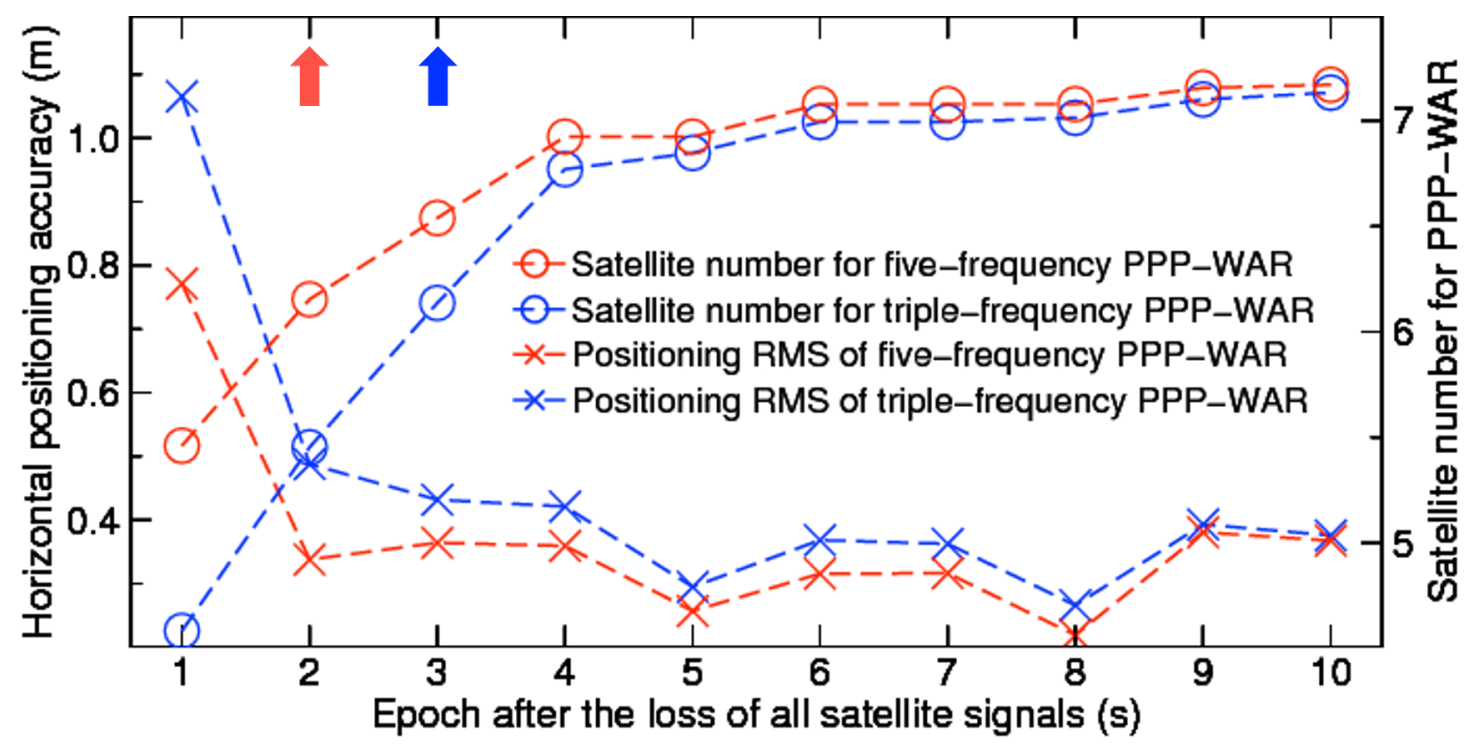

Figure 8 Instant horizontal positioning accuracy (m, crosses) of Galileo/BeiDou-3 five-frequency (E1/E5a/E5/E5b/E6 plus B1C/B3I/B2a) and triple-frequency (E1/E5a/E6 plus B1C/B3I/B2a) PPP- 
WAR at the first 10 epochs after the 12 loss-of-track events illustrated by the red crosses in Fig. 7.

We calculated a mean accuracy for each epoch over all 12 events. The mean satellite number (open circles) contributing to PPP-WAR for each epoch is also plotted referring to the right axis.

The solid red and blue arrows denote, respectively, the mean times to achieve successful fivefrequency and triple-frequency PPP-WAR solutions after the 12 total loss-of-track events. A "successful PPP-WAR solution" means that (extra-)wide-lane ambiguities for at least four satellites are resolved.

To investigate the resilience of instant five-frequency PPP-WAR against complex urban environments, two vehicle-borne multi-frequency Galileo/BeiDou-3 experiments (tracks A and B in Fig. 2) were carried out. The experiment along track A collected more than 4 BeiDou-3 satellites per epoch on average and came across only two overpasses marked as a and b (Fig. 2a). Along track B, however, there were five overpasses and one shaded passage marked as a, b, c, d, e and $\mathrm{f}$ in Fig. 2b, which severely interrupted satellite signals during the three laps of driving; only 1-3 BeiDou3 satellites could be observed for track B and hence BeiDou-3 only solutions were impossible. One-Hz instant PPP-WAR solutions were obtained using three strategies: Galileo/BeiDou-3, Galileo-only and BeiDou-3 only solutions. Short-baseline solutions using dual-frequency GPS/BeiDou-2 data were employed as truth benchmarks, where over $95 \%$ of ambiguities were fixed successfully.

Figure 6 shows the positioning accuracy of instant five-frequency PPP-WAR solutions for track A. It can be seen that even in an urban environment, positioning RMS of $0.22,0.23$ and $0.55 \mathrm{~m}$ for the east, north and up components are still achieved instantly when more than five Galileo satellites are observed, which are commensurate with the RMS reported in Table 5. Instant BeiDou-3 only PPP-WAR, in contrast, performs worse due largely to a poor satellite geometry when only 4-5 BeiDou-3 satellites were visible per epoch, but a positioning RMS of $0.33,0.34$ and $1.28 \mathrm{~m}$ is still attained over the one-hour driving. Encouragingly, the smallest positioning RMS of $0.17,0.21$ and $0.52 \mathrm{~m}$ for instant PPP-WAR can be achieved by 
integrating Galileo and BeiDou-3 multi-frequency data. In particular, the percentages of horizontal positions falling in $\pm 0.3 \mathrm{~m}$ are $45.3 \%, 74.6 \%$ and $84.2 \%$ for BeiDou-3 only, Galileo only and BeiDou-3/Galileo PPP-WAR, respectively. On the other hand, Fig. 7 shows the positioning accuracy of instant five-frequency PPP-WAR solutions in case of integrated Galileo E1/E5a/E5/E5b/E6 and BeiDou-3 B1C/B3I/B2a data from track B, where $3.28 \%$ of outliers caused by insufficient number of satellites (less than four) have been removed. Despite the 12 total signal interruptions, an accuracy of $0.23,0.24$ and $0.53 \mathrm{~m}$ (red traces) for the east, north and up components were still acquired, which could be improved to $0.14,0.12$ and $0.44 \mathrm{~m}$ (cyan traces) after multiepoch filtering. At this point, one should be reminded that multi-epoch filtering is always preferred whenever possible and achievable, while single-epoch or instant positioning resolves the harshest situation manifested by frequent satellite signal interruptions. This is also one of the important messages we aim at conveying from this study.

Furthermore, Fig. 8 shows the instant horizontal positioning accuracy at the first 10 epochs after the 12 total loss-of-track events. We calculated a mean accuracy for each epoch over the 12 events. The mean number of satellites contributing to PPPWAR is also plotted for each epoch. We find that five-frequency Galileo/BeiDou-3 (E1/E5a/E5/E5b/E6 and B1C/B3I/B2a) data can re-achieve successful PPP-WAR more rapidly than triple-frequency data (i.e., Galileo E1/E5a/E6 plus BeiDou-3 B1C/B3I/B2a) after total signal interruptions, as exemplified by the three typical snapshots listed in Fig. 9. In particular, as symbolized by the solid arrows in Fig. 8, the average time to recover PPP-WAR solutions from a total signal obstruction is $1 \mathrm{~s}$ using five-frequency data, in contrast to $2 \mathrm{~s}$ using triple-frequency data. In extreme cases, five-frequency data can ensure a rapid retrieval of PPP-WAR solutions $2 \mathrm{~s}$ earlier than that guaranteed by triple-frequency data. This $1-2 \mathrm{~s}$ of earlier retrieval is seemingly negligible, but actually of prime significance on account of the stringent life-safety standards required by self-driving vehicles. We demonstrate that higher data redundancy favored by more frequencies of signals increases the possibility of 
more satellites having at least two wide-lane observables resolved and thus contributing to PPP-WAR. This point can be corroborated by the mean satellite number plotted in Fig. 8, especially in the early stage of re-acquiring lost satellite signals.
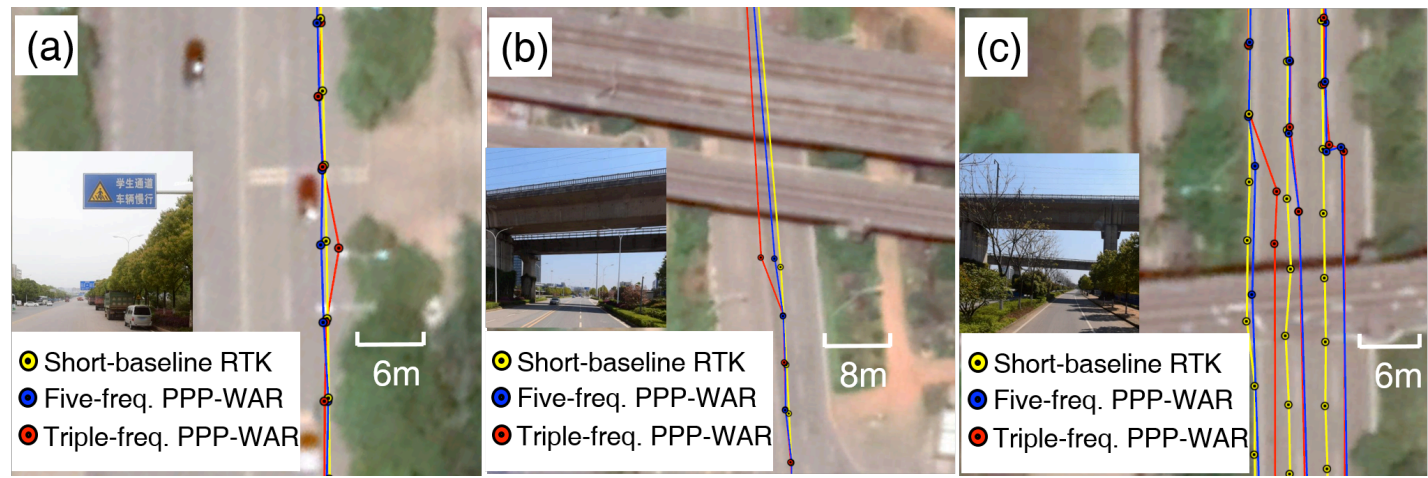

Figure 9 Three track B snapshots of the vehicle positioning overlaid on Google maps,

exemplifying the shaded passage $f$ and overpasses a and $c$ denoted in Fig. $2 b$. The yellow dots represent the benchmark short-baseline solutions, the blue dots represent the instant Galileo/BeiDou-3 (E1/E5a/E5/E5b/E6+B1C/B3I/B2a) five-frequency PPP-WAR solutions, and the red dots represent the instant triple-frequency Galileo/BeiDou-3 (E1/E5a/E6+B1C/B3I/B2a) PPP-WAR solutions. The colored dots overlaid along the lines denote the epochs of position estimates. We can find that five-frequency PPP-WAR can be usually more close to the benchmarks in case of GNSS-difficult situations, implying its higher availability of precise positioning.

\section{Discussion on instant PPP-WAR based on Galileo E6}

Table 6 has shown that instant Galileo-only PPP-WAR can achieve 20-cm horizontal positioning accuracy as long as the E6 signals are involved, while otherwise only a $30-\mathrm{cm}$ accuracy can be expected at most. Since the E6 carrier-phase noise level is similar to those of other Galileo signals (Basile et al., 2018), we speculate that it is the frequency spacing of E6 signals relative to others that makes E6-based PPP-WAR outperform all other counterparts without involving E6. Geng et al. (2019b) have demonstrated that, after resolving two wide-lane ambiguities among triple frequencies of signals, we are in essence constituting, no matter implicitly or explicitly, an 
ionosphere-free ambiguity-fixed wide-lane observable to enable decimeter-level positioning (e.g., Schaffrin and Bock, 1998; Teunissen, 1997). Though wide-lane ambiguity resolution can be carried out efficiently or even instantaneously, its shortcoming is that the noise of the ionosphere-free wide-lane observables can be amplified by over 100 times, degraded to a few decimeters and thus aggravate positioning precisions to the sub-meter level (Geng and Bock, 2013). In particular, the ionosphere-free wide-lane observable based on triple frequencies can be written as

$$
\begin{aligned}
L_{i, i f w}^{k}= & \frac{g_{s, j}}{g_{s, j}-1} \underbrace{\left(\frac{g_{s, 2}}{g_{s, 2}-1} L_{i, 1}^{k}-\frac{1}{g_{s, 2}-1} L_{i, 2}^{k}\right)}_{\text {Wide-lane combination }}- \\
& \frac{1}{g_{s, j}-1} \underbrace{\left(\frac{g_{s, j}}{g_{s, j}-g_{s, 2}} L_{i, 2}^{k}-\frac{g_{s, 2}}{g_{s, j}-g_{s, 2}} L_{i, j}^{k}\right)}_{\text {Extra-wide-lane combination }} \\
= & \frac{g_{s, 2} g_{s, j}}{\left(g_{s, 2}-1\right)\left(g_{s, j}-1\right)} L_{i, 1}^{k}- \\
& \frac{g_{s, j}}{\left(g_{s, 2}-1\right)\left(g_{s, j}-g_{s, 2}\right)} L_{i, 2}^{k}+ \\
& \frac{g_{s, 2}}{\left(g_{s, j}-1\right)\left(g_{s, j}-g_{s, 2}\right)} L_{i, j}^{k}
\end{aligned}
$$

where $L_{i, i f w}^{k}$ denotes the ionosphere-free wide-lane observable and $L_{i, 1}^{k}, L_{i, 2}^{k}$ and $L_{i, j}^{k}(j=3,4,5)$ represent raw triple-frequency observations. In this sense, we list representative ionosphere-free wide-lane combination observables in Table 7 for GPS, Galileo, BeiDou-2/3 and QZSS to inspect their noise amplification according to Eq. 13. Specifically, Table 7 exhibits the three combination coefficients and the final noise amplification factors. We find that the E6-based Galileo ionosphere-free wide-lane combination observable has the smallest noise amplification factor of 67.0 , which is less than one third of those for other Galileo combinations. The same amplification is also found for QZSS L1/L5/LEX signals since they have the same frequency assignments. This fact demonstrates that E6-based and LEX-based ionosphere-free wide-lane combination observables will have dramatically smaller noise to ensure that 
PPP-WAR can achieve much better positioning accuracy (cf. Table 6). Furthermore, we can find that BeiDou-3 also has such favorable ionosphere-free wide-lane combination observable based exclusively on B1C, B3I and B2a signals whose noise amplification factor is as low as 71.2. In fact, the B1C/B3I/B2a-enabled PPP-WAR solutions at station XIA1 (cf. Fig 1b) achieve 0.24 m positioning accuracy for the horizontal components, which is $20-30 \%$ better than those for the $\mathrm{B} 1 \mathrm{C} / \mathrm{B} 2 \mathrm{a} / \mathrm{B} 2 \mathrm{~b}$ and B1C/B3I/B2b PPP-WAR. We anticipate that BeiDou-3 instant PPP-WAR can be even better when more satellites are available in the near future. By integrating such lownoise wide-lane combinations from Galileo, BeiDou-3 and QZSS, it is promising to ensure a 10-20 cm positioning accuracy in the horizontal components using singleepoch PPP-WAR, once all constellations are fully operational.

Table 7 Combination coefficients of GPS/QZSS L1/L2/L5/LEX, Galileo E1/E5a/E5/E5b/E6 and BeiDou-2/3 B1C/B1I/B2a/B2b/B2I/B3I signals for the ionosphere-free wide-lane observables. The last column shows the noise amplification factor compared to raw carrier-phase observations. Bold numbers highlight the favorable combinations with low noise amplifications.

\begin{tabular}{|c|c|c|c|c|c|c|}
\hline GNSS & L1/E1/B1C/B1I & L2/E5a/B2a & L5/E5b/B2b/B2I & E5/B3I & E6/LEX & Factor \\
\hline GPS & 17.9 & -84.7 & 67.8 & & & 110.0 \\
\hline QZSS & 17.9 & -84.7 & 67.8 & & & 110.0 \\
\hline QZSS & 24.1 & 84.7 & & & -107.8 & 139.2 \\
\hline QZSS & 21.0 & & 33.9 & & -53.9 & 67.0 \\
\hline $\begin{array}{l}\text { Galileo } \\
\text { (E1/E5a/E5) }\end{array}$ & 16.2 & 226.1 & & -241.3 & & 331.0 \\
\hline $\begin{array}{l}\text { Galileo } \\
(\mathrm{E} 1 / \mathrm{E} 5 \mathrm{a} / \mathrm{E} 5 \mathrm{~b})\end{array}$ & 16.9 & 113.0 & -128.9 & & & 172.3 \\
\hline $\begin{array}{l}\text { Galileo } \\
(\text { E1/E5a/E6) }\end{array}$ & 21.0 & 33.9 & & & -53.9 & 67.0 \\
\hline
\end{tabular}


BeiDou-2

23.5

67.1

$-89.6$

114.4

(B1I/B2I/B3I)

BeiDou-3

16.9

113.0

$-128.9$

172.3

(B1C/B2a/B2b)

BeiDou-3

22.0

64.5

$-85.4$

109.3

(B1C/B3I/B2b)

BeiDou-3

20.3

37.7

(B1C/B3I/B2a)

\section{Conclusions and outlook}

In this study, we developed an extendable and flexible model for multi-frequency instant PPP-WAR, aiming at instantaneous $10-30 \mathrm{~cm}$ positioning accuracy using Galileo/BeiDou-3 signals. New-generation signals of BeiDou-3 were used in the data processing and their contribution to PPP-WAR was also investigated. All raw uncombined observations, whenever available, were injected into instant PPP-WAR after correction of satellite- and frequency-specific wide-lane phase biases. Wide-lane ambiguities were then converted at the normal equation level by choosing an arbitrary reference frequency, and were fixed simultaneously to integers using the integer leastsquares estimator to achieve single-epoch decimeter-level point positioning.

Data from 36 static stations in Australia spanning 30 days and 10 stations in China spanning 7 days were used to validate multi-frequency instant Galileo/BeiDou3 PPP-WAR. Uncombined wide-lane phase biases were estimated for each frequency with a high precision of 0.03 cycles and 0.05 cycles for Galileo and BeiDou-3, respectively. Moreover, Galileo phase biases show higher stability with a mean standard deviation of less than 0.006 cycles for $\mathrm{E} 5 \mathrm{a} / \mathrm{E} 5 \mathrm{~b} / \mathrm{E} 5$ signals and 0.016 cycles for E1 signal; in contrast, BeiDou-3 achieved a mean standard deviation of about 0.35 cycles for all frequencies due largely to the limited number of BeiDou-3 stations 
contributing to the phase bias estimation. We then carried out Galileo five-frequency PPP-WAR and an instant positioning accuracy of $0.10,0.11$ and $0.48 \mathrm{~m}$ for the east, north and up components, respectively, were achieved with more than 9 visible satellites per epoch. Comparatively, BeiDou-3 achieved an instant positioning accuracy of $0.16,0.23$ and $0.38 \mathrm{~m}$, which was relatively poor since there were only 56 visible satellites per epoch. Vehicle-borne experiments integrating Galileo and BeiDou-3 also showed an instant positioning accuracy of $0.17,0.21$ and $0.52 \mathrm{~m}$ for the east, north and up components, respectively, with 8-12 visible satellites per epoch. Furthermore, compared to triple-frequency PPP-WAR, five-frequency PPP-WAR solutions have higher positioning availability in case of the rapid recovery from total loss-of-track events. According to a vehicle-borne experiment in a challenging urban environment where satellite signals were totally interrupted 12 times within an hour, Galileo/BeiDou-3 five-frequency instant PPP-WAR can be recovered $1 \mathrm{~s}$ earlier on average than its triple-frequency counterpart, which is of major significance on account of the stringent life-safety standards for autonomous driving.

With integrated Galileo/BeiDou-3 observations in case of fully operational constellations, it is expected that a $10-20 \mathrm{~cm}$ instant positioning accuracy in either horizontal component can be achieved, promisingly even in a harsh GNSS-adverse environment. This performance is towards the satisfaction of the requirements for both high positioning accuracy and availability by driverless vehicles.

Acknowledgements This study is funded by National Science Foundation of China (41674033) and National Key Research and Development Program of China (2018YFC1504002). We thank IGS (International GNSS Service), ARGN (Australian Regional GNSS Network) and IGMAS for the multi-GNSS data and the high-quality satellite products. The computation work was accomplished on the high-performance computing facility of Wuhan University.

Author Contributions Statement JHG devised the project and the main conceptual 
ideas. JHG and JG worked out almost all of the technical details and performed the numerical calculations for the suggested experiments; JG analyzed the data; JHG and JG wrote the paper. All authors provided critical feedback and helped to shape the research, analysis and manuscript.

Data Availability Statement The raw GNSS data from ARGN are publicly available at ftp://ftp.ga.gov.au while the IGS products can be accessed at ftp://cddis.gsfc.nasa.gov. All data from IGMAS and the vehicle-borne experiments in the study are available from the authors upon request.

\section{References}

Basile F, Moore T, Hill C (2018) Analysis on the potential performance of GPS and Galileo Precise Point Positioning using simulated Real-Time Products. J Navig 72(1):19-33

Boehm J, Niell AE, Tregoning P, Schuh H (2006) The Global Mapping Function (GMF): a new empirical mapping function based on data from numerical weather model data. Geophys Res Lett 33, L07304.

de Jonge P. J. (1998) A processing strategy for the application of the GPS in networks. In: Publications on geodesy, 46, Netherlands Geodetic Commission, Delft, The Netherlands, pp225

Dong D, Bock Y (1989) Global positioning system network analysis with phase ambiguity resolution applied to crustal deformation studies in California. J Geophys Res 94(B4):3949-3966

Euler HJ, Schaffrin B (1990) On a measure of the discernibility between different ambiguity solutions in the static-kinematic GPS mode. In: Schwarz KP, Lachapelle G (eds) Kinematic systems in geodesy, surveying and remote sensing. Springer, New York, pp 285-295

Fantino M, Marucco G, Mulassano P, Pini M (2008) Performance analysis of MBOC, AltBOC and BOC modulations in terms of multipath effects on the carrier 
tracking loop within GNSS receivers. In: Proceedings of IEEE/ION PLANS, Monterey, CA, pp 369-376. doi: 10.1109/PLANS.2008.4570092

Feng Y, Li B (2010) Wide area real time kinematic decimeter positioning with multiple carrier GNSS signals. Sci China Ser D 53(5):731-740

Ge M, Gendt G, Rothacher M, Shi C, Liu J (2008) Resolution of GPS carrier-phase ambiguities in precise point positioning (PPP) with daily observations. J Geod 82(7):389-399

Geng J, Teferle FN, Meng X, Dodson AH (2011) Towards PPP-RTK: Ambiguity resolution in real-time precise point positioning. Adv Space Res 47(10):16641673

Geng J, Shi C, Ge M, Dodson AH, Lou Y, Zhao Q, Liu J (2012) Improving the estimation of fractional-cycle biases for ambiguity resolution in precise point positioning. J Geod 86(8):579-589

Geng J, Bock Y (2013) Triple-frequency GPS precise point positioning with rapid ambiguity resolution. J Geod 87(5):449-460

Geng J, Guo J, Chang H, Li X (2019a) Toward global instantaneous decimeter-level positioning using tightly coupled multi-constellation and multi-frequency GNSS. J Geod 93(7):977-991

Geng J, Guo J, Meng X, Gao K (2019b) Speeding up PPP ambiguity resolution using triple-frequency GPS/BeiDou/Galileo/QZSS data. J Geod doi: 10.1007/s00190019-01330-1

Geng J, Chen X, Pan Y, Zhao Q (2019c) A modified phase clock/bias model to improve PPP ambiguity resolution at Wuhan University. J Geod doi:10.1007/s00190-019-01301-6

Guo J, Geng J (2017) GPS satellite clock determination in case of inter-frequency clock biases for triple-frequency precise point positioning. J Geod 92(10):11331142

Guo J, Xin S (2019) Toward single-epoch 10-centimeter precise point positioning using Galileo E1/E5a and E6 signals. In: Proceedings of ION GNSS+ 2019, pp2870-2887, 16-20 Sept, Miami, FL 
Hatch R (2006) A new three-frequency, geometry-free technique for ambiguity resolution. In: Proceedings of ION GNSS 2006, 26-29 Sept, Fort Worth, TX, pp $309-316$

Kim J, Song, J, No H, Han D, Kim D, Park B, Kee C (2017) Accuracy improvement of DGPS for low-cost single-frequency receiver using modified Flächen Korrektur parameter correction. ISPRS Int. J. Geo-Inf. 6(7), 222

Laurichesse D, Banville S (2018) Innovation: instantaneous centimeter-level multifrequency precise point positioning. GPS World July 4, 2018 (www.gpsworld.com/innovation-instantaneous-centimeter-level-multi-frequencyprecise-point-positioning/)

Li B, Li Z, Zhang Z, Tan Y (2017) ERTK: extra-wide-lane RTK of triple-frequency GNSS signals. J Geod 91(9):1031-1047

Li P, Zhang X, Ge M, Schuh H (2018) Three-frequency BDS precise point positioning ambiguity resolution based on raw observables. J Geod doi:10.1007/ s00190$018-1125-3$

Lu M, Li W, Yao Z, Cui X (2019) Overview of BDS III new signals. Navigation $66(1): 19-35$

Nadarajah N, Khodabandeh A, Wang K, Choudhury M, Teunissen P (2018) MultiGNSS PPP-RTK: From large- to Small-Scale Networks. Sensors 18(4):1078

Odijk D, Zhang B, Khodabandeh A, Odolinski R, Teunissen P (2016) On the estimability of parameters in undifferenced, uncombined GNSS network and PPP-RTK user models by means of S-system theory. J Geod 90(1):15-44

Odolinski R, Teunissen PJG, Odijk D (2015) Combined BDS, Galileo, QZSS and GPS single-frequency RTK. GPS Solut 19(1):151-163

Prochniewicz D, Szpunar R, Brzezinski A (2016) Network-based stochastic model for instantaneous GNSS real-time kinematic positioning. J Surv Eng 142(4):05016004

Saastamoinen J (1973) Contribution to the theory of atmospheric refraction: refraction corrections in satellite geodesy. Bull Geod 107(1):13-34

Schaer S (2016) SINEX-Bias-Solution independent exchange format for GNSS biases 
version 1.00 (draft). In: IGS Workshop on GNSS biases, Bern, Switzerland, 5-6 Nov

Schaffrin B, Bock Y (1988) A unified scheme for processing GPS dual-band observations. Bull Geod 62:142-160

Schreiber M, Knöppel C, Franke U (2013) Laneloc: Lane marking based localization using highly accurate maps. In: Proceedings of the IEEE Intelligent Vehicles Symposium, Gold Coast, Australia, 23-26 June, pp 449-454.

Stephenson S, Meng X, Moore T, Baxendale A, Edwards T (2013) Network RTK for intelligent vehicles: Accurate, reliable, available, continuous positioning for cooperative driving. GPS World 24(2):61-67

Teunissen PJG (1995) The least-squares ambiguity decorrelation adjustment: a method for fast GPS integer ambiguity estimation. J Geod 70(1-2):65-82

Teunissen PJG (1997) On the GPS widelane and its decorrelating property. J Geod 71(9):577-587

Teunissen PJG, Odolinski R, Odijk D (2014) Instantaneous BeiDou+GPS RTK positioning with high cut-off elevation angles. J Geod 88(4):335-350

Teunissen PJG, Khodabandeh A (2015) Review and principles of PPP-RTK methods. J Geod 89(3):217-240

Wang K, Khodabandeh A, Teunissen PJG (2018) Five-frequency Galileo longbaseline ambiguity resolution with multipath mitigation. GPS Solut 22:75

Zhang X, Wu M, Liu W, Li X, Yu S, Lu C, Wickert J (2017) Initial assessment of the COMPASS/BeiDou-3: new-generation navigation signals. J Geod doi: $10.1007 / \mathrm{s} 00190-017-1020-3$

Zhao Q, Wang C, Guo J, Wang B, Liu J (2017) Precise orbit and clock determination for BeiDou-3 experimental satellites with yaw attitude analysis. GPS Solut 22(1):4.

Zumberge JF, Heflin MB, Jefferson DC, Watkins MM, Webb FH (1997) Precise point positioning for the efficient and robust analysis of GPS data from large networks. J Geophys Res 102(B3):5005-5017 